\title{
Entanglement between pairing and screening in the Gorkov-Melik-Barkhudarov correction to the critical temperature throughout the BCS-BEC crossover
}

\author{
L. Pisani ${ }^{1}$ A. Perali, ${ }^{2}$ P. Pieri ${ }^{1,3, *}$ and G. Calvanese Strinati ${ }^{1,3, \dagger}$ \\ ${ }^{1}$ School of Science and Technology, Physics Division, Università di Camerino, 62032 Camerino, Italy \\ ${ }^{2}$ School of Pharmacy, Physics Unit, Università di Camerino, 62032 Camerino, Italy \\ ${ }^{3}$ INFN, Sezione di Perugia, 06123 Perugia, Italy
}

(Received 12 December 2017; published 30 January 2018)

\begin{abstract}
The problem of the theoretical description of the critical temperature $T_{c}$ of a Fermi superfluid dates back to the work by Gorkov and Melik-Barkhudarov (GMB), who addressed it for a weakly coupled (dilute) superfluid in what would today be referred to as the (extreme) BCS (weak-coupling) limit of the BCS-BEC crossover. The point made in this context by GMB was that particle-particle (pairing) excitations, which are responsible for superfluidity to occur below $T_{c}$, and particle-hole excitations, which give rise to screening also in a normal system, get effectively disentangled from each other in the BCS limit, thus yielding a reduction by a factor of 2.2 of the value of $T_{c}$ obtained when neglecting screening effects. Subsequent work on this topic, that was aimed at extending the original GMB argument away from the BCS limit with diagrammatic methods, has tout court kept this disentangling between pairing and screening throughout the BCS-BEC crossover, without realizing that the conditions for it to be valid are soon violated away from the BCS limit. Here, we reconsider this problem from a more general perspective and argue that pairing and screening are intrinsically entangled with each other along the whole BCS-BEC crossover but for the BCS limit considered by GMB, with the particle-hole excitations soon transmuting into particle-particle excitations away from this limit. We substantiate our argument by performing a detailed numerical calculation of the GMB diagrammatic contribution suitably extended to the whole BCS-BEC crossover, where the full wave-vector and frequency dependence occurring in the repeated in-medium two-particle scattering is duly taken into account. Our numerical calculations are tested against analytic results available in both the BCS and BEC limits, and the contribution of the GMB diagrammatic term to the scattering length of composite bosons in the BEC limit is highlighted. We calculate $T_{c}$ throughout the BCS-BEC crossover and find that it agrees quite well with quantum Monte Carlo calculations and experimental data available in the unitarity regime.
\end{abstract}

DOI: 10.1103/PhysRevB.97.014528

\section{INTRODUCTION}

In the original work by Bardeen, Cooper, and Schrieffer (BCS) on the theory of superconductivity [1], the attractive interparticle interaction of strength $U_{0}$ acting between opposite-spin fermions was considered to affect an energy region about the Fermi surface with width of the order of the Debye frequency $\omega_{D}$ (we set $\hbar=1$ throughout). This led to a critical temperature for the onset of superconductivity, of the form

$$
k_{B} T_{c}^{\mathrm{BCS}}=\frac{2 e^{\gamma} \omega_{D}}{\pi} \exp \left\{-1 /\left(N_{0}\left|U_{0}\right|\right)\right\},
$$

where $N_{0}=m k_{F} /\left(2 \pi^{2}\right)$ is the density of states at the Fermi level per spin component $\left[m\right.$ being the fermion mass and $k_{F}=$ $\left(3 \pi^{2} n\right)^{1 / 3}$ the Fermi wave vector associated with the particle density $n$ ], $e$ the Euler number, and $k_{B}$ and $\gamma$ the Boltzmann and Euler constants, respectively (with $e^{\gamma} \simeq 1.781$ ).

Soon after the BCS paper, Gorkov and Melik-Barkhudarov (GMB) considered the phenomenon of superfluidity in a dilute

\footnotetext{
*pierbiagio.pieri@unicam.it

† giancarlo.strinati@unicam.it
}

(neutral) Fermi gas [2]. This physical system has the advantage over the BCS model that the effects of the interparticle interaction can be expressed entirely in terms of the scattering length $a_{F}$ of the two-fermion problem in vacuum, thereby leaving aside the uncertainties related to the strength $U_{0}$ and the cutoff $\omega_{D}$. The dilute Fermi gas was originally considered by Galitskii for the case of a repulsive interparticle interaction, for which $a_{F}>0$ and $k_{F} a_{F} \ll 1[3]$. To deal with the phenomenon of superfluidity, GMB extended this treatment to the case of an attractive interparticle interaction, for which $a_{F}<0$ and $k_{F}\left|a_{F}\right| \ll 1$. Nowadays, full experimental control of the fermionic scattering length $a_{F}$ can be achieved with ultracold Fermi gases with an attractive interparticle interaction [4].

Having disposed of the quantities $U_{0}$ and $\omega_{D}$ with their associated uncertainties, the critical temperature for a dilute Fermi gas can still be obtained within the BCS mean-field decoupling, leading to the expression

$$
k_{B} T_{c}^{\mathrm{BCS}}=\frac{8 e^{\gamma} E_{F}}{\pi e^{2}} \exp \left\{\pi /\left(2 k_{F} a_{F}\right)\right\},
$$

where $E_{F}=k_{F}^{2} /(2 m)$ is the Fermi energy. [Note that expression (2) can be formally obtained from the original BCS expression [Eq. (1)] with the replacements $\omega_{D} \rightarrow 4 E_{F} / e^{2}$ and $U_{0} \rightarrow 4 \pi a_{F} / m$.] What GMB then realized was that, owing to 
the exponential dependence of the BCS expression [Eq. (2)] for $T_{c}$, if additional terms in the small parameter $k_{F} a_{F}$ could be introduced in the exponent such that $\left(k_{F} a_{F}\right)^{-1} \rightarrow\left(k_{F} a_{F}\right)^{-1}+$ $b+c\left(k_{F} a_{F}\right)+\cdots$, where $b$ and $c$ are constants, the constant $b$ would modify the BCS prefactor of $T_{c}$ by a finite amount even in the (extreme) weak-coupling limit when $k_{F} a_{F} \rightarrow 0^{-}$. To obtain the constant $b$, GMB considered a correction to the BCS instability of the normal phase that occurs when $T_{c}$ is approached from above. This instability can be obtained diagrammatically in terms of the "series of ladder graphs" in the particle-particle channel [5] and yields correspondingly for $T_{c}$ the BCS mean-field result (2) as obtained when $T_{c}$ is approached from below. For this reason, the GMB correction is sometimes referred to as "beyond-mean-field approximation." The end result of the GMB calculation for $T_{c}$ was a reduction of expression (2) of $T_{c}$ by the factor $(4 e)^{1 / 3} \simeq 2.2$. This result was obtained by performing, in practice, all wave-vector integrations contained in the diagrammatic expressions near the Fermi surface, with the assumption that the fermionic chemical potential $\mu$ coincides with $E_{F}$.

Later on, the GMB effect on $T_{c}$ was interpreted on physical grounds in terms of "polarization" effects of the medium occurring in the particle-hole channel due to particle-hole excitations across the Fermi surface [6], and attempts along these lines were also made to extend the GMB result to the high-density regime [7]. In all cases, these calculations were still limited to the (weak-coupling) BCS limit where the noninteracting Fermi surface is only slightly perturbed by the interparticle attraction. In this limit (as we comment more extensively below), the primary BCS instability occurring in the particle-particle channel and the polarization effects of the medium occurring in the particle-hole channel get effectively disentangled from each other (with an accompanying large reduction in the computational effort for determining their effect on $T_{c}$ ). Extensions of the GMB result were also considered for lower dimensionality [8], for mixtures of twocomponent fermionic atoms with different masses [9], for imbalanced spin populations in quasi two dimensions [10], and for lattice models [11], but in all cases still adopting the standard approximations that apply to the BCS regime.

With the advent of the experiments with ultracold Fermi gases and the related study of the BCS-BEC crossover, however, the question has naturally arisen about what would be the effect of the GMB correction when departing from the BCS limit. In particular, it is of interest to assess whether this correction may still yield significant effects at the unitary limit $\left(k_{F} a_{F}\right)^{-1}=0$ where a remnant Fermi surface is still active [12]. By the BCS-BEC crossover, there occurs a progressive reduction of the size of the fermionic pairs, ranging from the large size of strongly overlapping Cooper pairs in the BCS limit of weak interparticle attraction, to the small size of nonoverlapping composite bosons in the BEC limit of a strong interparticle attraction, across the intermediate unitary limit where the size of the pairs is comparable with the average interparticle distance. This crossover is driven by the coupling parameter $\left(k_{F} a_{F}\right)^{-1}$, which ranges from $\left(k_{F} a_{F}\right)^{-1} \lesssim-1$ in the weak-coupling (BCS) regime when $a_{F}<0$, to $\left(\hat{k}_{F} a_{F}\right)^{-1} \gtrsim$ +1 in the strong-coupling (BEC) regime when $a_{F}>0$, across the unitary limit when $\left|a_{F}\right|$ diverges. Correspondingly, the fermionic chemical potential $\mu$ ranges from $E_{F}$ in the BCS limit to $-\left(2 m a_{F}^{2}\right)^{-1}$ in the BEC limit, with a large variation occurring in between these two limits.

In this context, when dealing diagrammatically with the GMB correction to $T_{c}$ throughout the BCS-BEC crossover, the effective disentangling between particle-particle and particlehole channels mentioned above, which should confidently apply to the BCS limit only, was instead carried over tout court to the whole crossover $[13,14]$. In practice, this was simply done by calculating the particle-hole bubble associated with particle-hole excitations (suitably averaged over the Fermi sphere, like in the original GMB calculation), but now with a chemical potential that spans the whole crossover and thus is no longer equal to $E_{F}$ (apart from minor differences resulting from the way the chemical potential itself is calculated $[13,14])$. Recently, extensions along these lines were also considered to investigate the GMB correction when including the effect of the Rashba spin-orbit coupling in two-dimensional Fermi gases [15]. A completely different approach was instead followed in Ref. [16], where particle-particle and particle-hole bubbles were included simultaneously in the framework of the functional-renormalization-group approach.

The purpose of this paper is to analyze and settle the question of entanglement vs disentanglement between pairing and screening in the GMB correction to the critical temperature throughout the whole BCS-BEC crossover, by a careful analysis of the relevant many-body diagrammatic structure of the theory. Our analysis combines both extensive numerical calculations applied to the whole crossover and analytic results in the BCS and BEC limits, and avoids at the outset the approximations introduced originally by GMB. We show that these approximations, which entail an effective disentanglement between pairing (in the particle-particle channel) and screening (in the particle-hole channel), hold only in the BCS limit where the particle-particle propagator is approximately constant over a large sector of the wave-vector and frequency domain. Away from the BCS limit, the particle-particle (pair) propagator acquires instead a progressively marked dependence on wave vector and frequency and the GMB disentanglement between pairing and screening no longer holds. This feature makes the numerical calculation of the GMB correction to the critical temperature quite more involved than those reported, e.g., in Refs. [13,14], where the GMB disentanglement was assumed to hold for the whole crossover. In addition, for a realistic calculation of the critical temperature throughout the whole BCS-BEC crossover, we have combined the GMB correction, which evolves from the BCS to the BEC limits, with another correction which instead evolves in the opposite direction from the BEC to the BCS limits, since it was conceived in Ref. [17] to improve on the description of composite bosons in the BEC limit, at the level of the Popov approximation for pointlike bosons [18]. This combined Popov-GMB calculation for the critical temperature throughout the whole BCS-BEC crossover will yield a quite good comparison with quantum Monte Carlo data over the whole coupling range for which they are available and with experimental data at unitarity. It turns out, however, that the associated value of the chemical potential at unitarity does not match the corresponding experimental value. We attribute this difference to the lack of self-consistency in the fermionic propagators that underlie our theoretical approach, and argue at the same time that inclusion of self-consistency 
would not affect the good result obtained for the critical temperature.

Finally, it is relevant to mention that interest in the effects of medium polarization has also arisen in the context of low-density neutron matter, to the extent that these effects affect the value of the pairing gap in the superfluid phase at zero temperature $[19,20]$. Actually, it was already shown in the original GMB paper [2] that their beyond-mean-field approximation also renormalizes the value of the pairing gap at zero temperature with respect to the BCS value, in quite the same way that it does for the value of the critical temperature. The question of the entanglement between pairing and screening away from the weak-coupling (BCS) limit, that we discuss in detail in this paper for the critical temperature, is then expected to be relevant for the pairing gap as well and will be considered in future work.

The plan of the paper is as follows. In Sec. II we set up a diagrammatic approach to pairing fluctuations above $T_{c}$, which includes the GMB contribution in a form that can be extended to the whole BCS-BEC crossover. In this way, we show that in the BCS limit pairing and screening get effectively disentangled from each other, thus recovering the original GMB result. We also show, however, that this disentanglement cannot be sustained away from the BCS limit. To this end, we calculate analytically how the GMB correction evolves toward the BEC limit, showing that in this limit it yields a significant contribution to the scattering length for composite bosons. We further discuss the need to introduce the Popov correction mentioned above. In Sec. III we report on the numerical calculation of the Popov and GMB corrections and show how the relevant quantities in this context behave across the BCS-BEC crossover. We also discuss a (partial) self-consistent procedure on the pair propagator which is required for the calculation of $T_{c}$, owing to the fact that this propagator diverges upon approaching the superfluid phase when lowering the temperature from the normal phase. The numerical results for the Popov and GMB corrections are tested against their analytic expressions from Sec. II in the BCS and BEC limits, and our results for $T_{c}$ throughout the BCS-BEC crossover are compared with the quantum Monte Carlo and experimental data available in the unitary regime. Section IV gives our conclusions and sets up future perspectives of our approach. Appendix A discusses in detail the way the numerical calculations of the Popov and GMB corrections have been implemented in practice, in view of the highly nontrivial task of including the full wave-vector and frequency dependence of the pair propagators that enter these corrections. Finally, Appendix B shows how the Popov and GMB corrections contribute to the value of the scattering length for composite bosons that form in the BEC limit.

\section{EXTENDING THE GMB CONTRIBUTION THROUGHOUT THE BCS-BEC CROSSOVER}

In this section, we show how the GMB contribution has to be handled away from the BCS (weak-coupling) limit of the BCS-BEC crossover, in the normal phase above the critical temperature $T_{c}$. To this end, we begin by discussing the essential aspects of the many-body diagrammatic theory for a dilute Fermi gas with an attractive interparticle interaction, which are relevant to this problem. We are explicitly concerned with determining the value of $T_{c}$ throughout the BCS-BEC crossover, by resting on a minimal set of diagrammatic terms which include the GMB contribution. In the following, both the reduced Planck constant $\hbar$ and the Boltzmann constant $k_{B}$ are set equal to unity.

\section{A. Brief summary about pairing fluctuations in the normal phase above $T_{c}$}

A dilute Fermi gas is characterized by the fact that the range of the interparticle interaction is much smaller than the average interparticle distance, such that the interaction can be taken of the contact type $v_{0} \delta\left(\mathbf{r}-\mathbf{r}^{\prime}\right)$ acting between opposite-spin fermions. In the following, only the attractive case $v_{0}<0$ is considered and equal spin populations are taken.

The choice of a contact potential entails the introduction of an ultraviolet cutoff $k_{0}$ in the otherwise divergent integrals over the wave vector $\mathbf{k}$. The two quantities $v_{0}$ and $k_{0}$ can be combined together by resorting to the two-body problem in vacuum, whereby the fermionic scattering length $a_{F}$ is obtained from the relation [21]

$$
\frac{m}{4 \pi a_{F}}=\frac{1}{v_{0}}+\int_{|\mathbf{k}| \leqslant k_{0}} \frac{d \mathbf{k}}{(2 \pi)^{3}} \frac{m}{\mathbf{k}^{2}} .
$$

A suitable regularization procedure can be introduced at this point which eliminates further reference to $v_{0}$ and $k_{0}$, by taking the limits $v_{0} \rightarrow 0^{-}$and $k_{0} \rightarrow \infty$ simultaneously such that $a_{F}$ remains fixed at a desired value.

The above procedure is especially relevant when dealing with ultracold Fermi gases, for which $a_{F}$ can be experimentally controlled [4]. From the theoretical side, this regularization procedure somewhat simplifies the structure of the many-body diagrammatic theory. This is because a given diagram, which can be drawn for finite $v_{0}$, survives the limit $v_{0} \rightarrow 0^{-}$provided there occurs a compensating ultraviolet divergence for $k_{0} \rightarrow$ $\infty$. It turns out then that in this way the bare interaction $v_{0}$ is everywhere replaced by the effective interaction (or bare pair propagator) $\Gamma_{0}(Q)$ for opposite-spin fermions, where $Q=$ $\left(\mathbf{Q}, \Omega_{\nu}\right)$ is a four-vector with bosonic Matsubara frequency $\Omega_{v}=2 \pi \nu T$ ( $\nu$ integer). The pair propagator, depicted in Fig. 1(a), corresponds to an infinite sequence of two-body scattering events in the particle-particle channel (or ladder diagrams) and is given by the expression

$$
\Gamma_{0}(Q)=\frac{-v_{0}}{1+v_{0} \chi_{\mathrm{pp}}(Q)}=-\frac{1}{\frac{m}{4 \pi a_{F}}+R_{\mathrm{pp}}(Q)} .
$$

Here,

$$
\chi_{\mathrm{pp}}(Q)=\int \frac{d \mathbf{k}}{(2 \pi)^{3}} T \sum_{n} G_{0}\left(\mathbf{k}+\mathbf{Q}, \omega_{n}+\Omega_{v}\right) G_{0}\left(-\mathbf{k},-\omega_{n}\right)
$$

is the particle-particle bubble, where $G_{0}\left(\mathbf{k}, \omega_{n}\right)=\left(i \omega_{n}-\right.$ $\left.\xi_{\mathbf{k}}\right)^{-1}$ is the bare fermionic single-particle propagator with $\xi_{\mathbf{k}}=$ $\mathbf{k}^{2} /(2 m)-\mu$ ( $\mu$ being the chemical potential) and fermionic Matsubara frequency $\omega_{n}=(2 n+1) \pi T$ ( $n$ integer), and

$$
\begin{aligned}
R_{\mathrm{pp}}(Q) & =\chi_{\mathrm{pp}}(Q)-\int \frac{d \mathbf{k}}{(2 \pi)^{3}} \frac{m}{\mathbf{k}^{2}} \\
& =\int \frac{d \mathbf{k}}{(2 \pi)^{3}}\left(\frac{1-f\left(\xi_{\mathbf{k}+\mathbf{Q}}\right)-f\left(\xi_{\mathbf{k}}\right)}{\xi_{\mathbf{k}+\mathbf{Q}}+\xi_{\mathbf{k}}-i \Omega_{v}}-\frac{m}{\mathbf{k}^{2}}\right)
\end{aligned}
$$




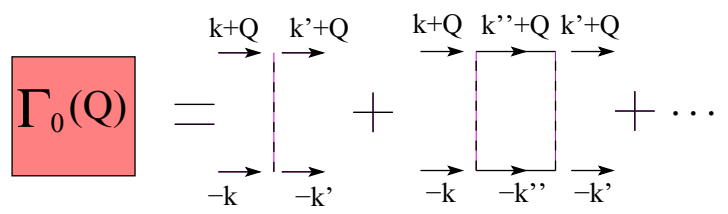

(a)

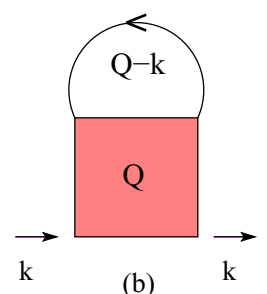

FIG. 1. Diagrammatic representation of (a) the bare pair propagator $\Gamma_{0}$ for opposite-spin fermions and (b) the single-particle fermionic self-energy $\Sigma$ obtained from $\Gamma_{0}$ within the (non-self-consistent) $t$-matrix approximation. Solid and dashed lines represent the bare fermionic single-particle propagator $G_{0}$ and the interaction potential $v_{0}$, respectively, while $Q(k)$ are bosonic (fermionic) four-vectors. Here and in the following, the upper (lower) line of $\Gamma_{0}$ corresponds to an up (down) fermionic spin.

is the regularized version of the particle-particle bubble obtained with the help of Eq. (3) $\left[f(E)=(\exp \{E / T\}+1)^{-1}\right.$ being the Fermi function].

Accordingly, the pair propagator $\Gamma_{0}$ represents a building block of the diagrammatic theory in the normal phase above $T_{c}$. In particular, the simplest possible fermionic self-energy is obtained in terms of a single $\Gamma_{0}$, as depicted in Fig. 1(b) and given by the expression

$$
\Sigma(k)=-\int \frac{d \mathbf{Q}}{(2 \pi)^{3}} T \sum_{\nu} \Gamma_{0}(Q) G_{0}(Q-k)
$$

with the fermionic four-vector notation $k=\left(\mathbf{k}, \omega_{n}\right)$. The choice of Eq. (7) for $\Sigma$ corresponds to the so-called nonself-consistent $t$-matrix approximation. This self-energy was originally considered by Nozières and Schmitt-Rink (NSR) to account for the correct behavior of the BCS-BEC crossover in the BEC limit at finite temperature [22]. In the following, we adopt the approach of Ref. [23] and use the self-energy [Eq. (7)] to obtain the (dressed) fermionic single-particle propagator $G(k)=\left[G_{0}(k)^{-1}-\Sigma(k)\right]^{-1}$, in terms of which the total fermionic density

$$
n=2 \int \frac{d \mathbf{k}}{(2 \pi)^{3}} T \sum_{n} e^{i \omega_{n} \eta} G(k)
$$

(where $\eta=0^{+}$) can be calculated to obtain the chemical potential for given temperature and coupling. In the original NSR approach [22], however, the density was derived from Eq. (8) with the additional approximation of expressing $G$ at first order in $\Sigma$, thereby writing $G(k) \simeq G_{0}(k)+G_{0}(k) \Sigma(k) G_{0}(k)$.

\section{B. Thouless criterion}

The pair propagator $\Gamma_{0}(Q)$ plays also the role of signaling the insurgence of the broken-symmetry (superfluid) phase, when the temperature is lowered down to the critical temperature $T_{c}$. This is because the sequence of ladder diagrams on which the pair propagator is built diverges for $Q=0$, thereby manifesting that, upon approaching $T_{c}$, pairing fluctuations are able to organize themselves over a progressively larger spatial distance. The critical temperature is thus determined by the following condition (known also as the Thouless criterion [24]):

$$
\begin{aligned}
-\Gamma_{0}(Q= & \left.0 ; T_{c}, \mu_{c}\right)^{-1}=\frac{m}{4 \pi a_{F}} \\
& +\int \frac{d \mathbf{k}}{(2 \pi)^{3}}\left(\frac{\tanh \left(\xi_{\mathbf{k}} / 2 T_{c}\right)}{2 \xi_{\mathbf{k}}}-\frac{m}{\mathbf{k}^{2}}\right)=0,
\end{aligned}
$$

obtained upon setting $Q=0$ in expression (4) and with the thermodynamic variables explicitly indicated. This expression formally coincides with that obtained within the BCS meanfield approximation when $T_{c}$ is reached from below. Care should, however, be exerted about the value of $\mu_{c}=\mu\left(T_{c}\right)$ that enters Eq. (9).

For later purposes, it is relevant to obtain analytically the expression of $T_{c}$ in the BCS (weak-coupling) limit within the non-self-consistent $t$-matrix approximation of Eqs. (7) and (8). To this end, the integral on the right-hand side of Eq. (9) can be calculated analytically under the typical weak-coupling approximation $T_{c} \ll \mu$. One obtains

$$
\begin{aligned}
& \int \frac{d \mathbf{k}}{(2 \pi)^{3}}\left(\frac{\tanh \left(\xi_{\mathbf{k}} / 2 T_{c}\right)}{2 \xi_{\mathbf{k}}}-\frac{m}{\mathbf{k}^{2}}\right) \\
& \simeq \frac{(2 m)^{3 / 2} \sqrt{\mu}}{4 \pi^{2}}\left[\ln \left(\frac{8 \mu e^{\gamma}}{\pi T_{c}}\right)-2\right] .
\end{aligned}
$$

Entering this result into the Thouless criterion (9) yields

$$
T_{c} \simeq \frac{8 e^{\gamma} \mu}{\pi e^{2}} \exp \left\{\frac{\pi}{2 k_{F} a_{F}} \sqrt{\frac{E_{F}}{\mu}}\right\} .
$$

Within the non-self-consistent $t$-matrix approximation (as well as in the NSR approach), to the leading order in the small parameter $k_{F}\left|a_{F}\right|$ (where $a_{F}<0$ ) the chemical potential (for $T \ll T_{F}$, as is the case in weak coupling close to $T_{c}$ ) is given by

$$
\mu=E_{F}\left[1+\frac{4}{3 \pi} k_{F} a_{F}+\cdots\right],
$$

such that $\pi \sqrt{E_{F} / \mu} /\left(2 k_{F} a_{F}\right) \simeq \pi /\left(2 k_{F} a_{F}\right)-1 / 3$ in the exponent of Eq. (11). This results in a "spurious" factor $e^{-1 / 3}$ to appear on the right-hand side of Eq. (11) with respect to the expected BCS (weak-coupling) result (2). This shortcoming can be remedied by introducing a partial degree of self-consistency in the non-self-consistent $t$-matrix approximation through a constant self-energy (mean-field) shift $\Sigma_{0} \simeq 2 \pi a_{F} n / m$ [23], such that $\mu-\Sigma_{0} \simeq E_{F}$ again at the leading order in $k_{F}\left|a_{F}\right|$. In this way, expression (2) for $T_{c}$ is correctly recovered.

In the following, a partial degree of self-consistency is introduced in the non-self-consistent $t$-matrix approximation, not only in the BCS (weak-coupling) limit but also throughout the whole BCS-BEC crossover, by relying on the approach of Ref. [17]. This approach (to be discussed next) was originally conceived to improve on the description of composite bosons (dimers) that form in the BEC limit with respect to the 


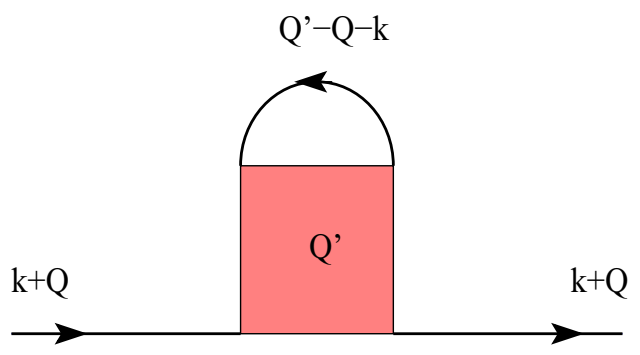

(a)

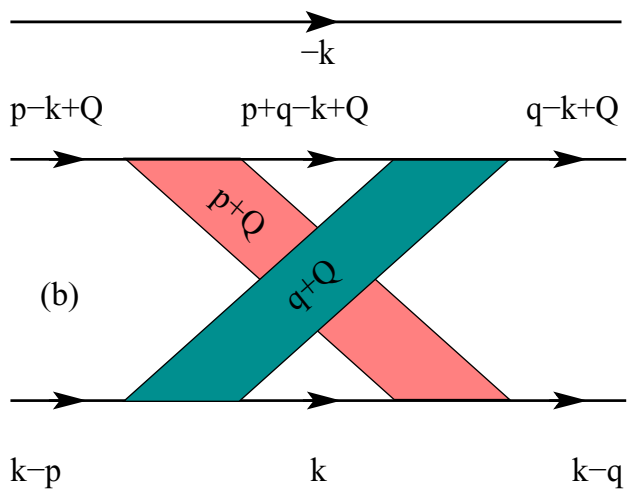

FIG. 2. Diagrammatic representation of (a) the Popov bosoniclike self-energy $\Sigma_{\text {Popov }}^{\mathrm{B}}$, obtained by dressing the upper fermionic line in the particle-particle channel (an analogous dressing occurs for the lower fermionic line), and (b) the GMB bosoniclike self-energy $\Sigma_{\mathrm{GMB}}^{\mathrm{B}}$. In both cases, upper and lower fermionic lines correspond to opposite spins. By the present approach, $\Sigma_{\mathrm{Popov}}^{\mathrm{B}}$ and $\Sigma_{\mathrm{GMB}}^{\mathrm{B}}$ represent bosoniclike self-energy insertions to the pair propagator $\Gamma_{0}$.

non-self-consistent $t$-matrix approximation by introducing a kind of mean-field interaction also between the otherwise noninteracting composite bosons.

\section{Popov contribution}

In Ref. [17], an approximation for the BCS-BEC crossover was devised, which in the strong-coupling limit of the fermionic attraction would extend the Popov description from pointlike [18] to composite bosons. In this way, a residual interaction among composite bosons survives even when the condensate disappears above the critical temperature. Under these circumstances, the bare pair propagator $\Gamma_{0}$ gets dressed through the bosoniclike self-energy depicted in Fig. 2(a), whose analytic expression reads [25]

$$
\begin{aligned}
\Sigma_{\text {Popov }}^{\mathrm{B}}(Q)= & -2 \int \frac{d \mathbf{k}}{(2 \pi)^{3}} T \sum_{n} \int \frac{d \mathbf{Q}^{\prime}}{(2 \pi)^{3}} T \sum_{v^{\prime}} \\
& \times G_{0}(k+Q)^{2} G_{0}(-k) G_{0}\left(Q^{\prime}-Q-k\right) \Gamma_{0}\left(Q^{\prime}\right),
\end{aligned}
$$

where the factor of 2 accounts for the dressing of both upper and lower fermionic lines. In the following, we limit ourselves to consider the case $Q=0$, whereby we set $\Sigma_{\text {Popov }}^{\mathrm{B}}=$ $\Sigma_{\text {Popov }}^{\mathrm{B}}(Q=0)$.
Both the BCS (weak-coupling) and BEC (strong-coupling) limits of $\Sigma_{\text {Popov }}^{\mathrm{B}}$ can be calculated analytically. In particular, the BCS limit is obtained as follows. One begins by approximating $\Gamma_{0}\left(Q^{\prime}\right) \simeq-4 \pi a_{F} / m$ in Eq. (13), such that

$$
\int \frac{d \mathbf{Q}^{\prime}}{(2 \pi)^{3}} T \sum_{\nu^{\prime}} G_{0}\left(Q^{\prime}-k\right) \Gamma_{0}\left(Q^{\prime}\right) \simeq-\frac{4 \pi a_{F}}{m} \frac{n_{0}(T, \mu)}{2},
$$

where $n_{0}(T, \mu)$ is the density of a system of noninteracting fermions with Fermi energy $\mu$ at temperature $T$. Accordingly, for $T \ll T_{F}$ we can write $n_{0}(T, \mu)=k_{\mu}^{3} /\left(3 \pi^{2}\right)$, with the notation $\mu=k_{\mu}^{2} /(2 m)$ (with $\mu>0$ ) [23]. The remaining factor on the right-hand side of Eq. (13) can be calculated by noting that

$$
\begin{aligned}
& \int \frac{d \mathbf{k}}{(2 \pi)^{3}} T \sum_{n} G_{0}(k)^{2} G_{0}(-k) \\
& =-\frac{1}{2} \frac{\partial}{\partial \mu} \int \frac{d \mathbf{k}}{(2 \pi)^{3}} T \sum_{n} G_{0}(k) G_{0}(-k) \\
& =-\frac{1}{2} \frac{\partial}{\partial \mu} R_{p p}(Q=0) \\
& \simeq-\frac{1}{2} \frac{(2 m)^{3 / 2}}{8 \pi^{2} \sqrt{\mu}}\left\{\left[\ln \left(\frac{8 \mu e^{\gamma}}{\pi T}\right)-2\right]+2\right\} \simeq \frac{m^{2}}{8 \pi a_{F} k_{\mu}^{2}} .
\end{aligned}
$$

To obtain this result, we have made use of definition (6) for the regularized particle-particle bubble (with $Q=0$ ) and of its approximate expression [Eq. (10)] valid in the BCS limit, as well as of the result $\ln (\mu / T) \simeq-\pi /\left(2 k_{\mu} a_{F}\right)$ obtained by the Thouless criterion [Eq. (9)] in the absence of the Popov correction by assuming that $T$ is of the order of the critical temperature $T_{c}$.

Entering the results Eqs. (14) and (15) in Eq. (13) with $Q=0$, we obtain eventually in the BCS limit

$$
\Sigma_{\text {Popov }}^{\mathrm{B}} \simeq \frac{m k_{\mu}}{6 \pi^{2}}=\frac{1}{3} \frac{(2 m)^{3 / 2} \sqrt{\mu}}{4 \pi^{2}} .
$$

Here, the expression on the right-hand side makes evident the presence of a factor $1 / 3$, which is required to eliminate the "spurious" factor $e^{-1 / 3}$ in the expression of the critical temperature as noted after Eq. (12).

To this end, we modify the original Thouless criterion [Eq. (9)] by including the Popov contribution, in the form

$$
\Gamma_{0}\left(Q=0 ; T_{c}, \mu_{c}\right)^{-1}-\Sigma_{\text {Popov }}^{\mathrm{B}}(Q=0)=0 .
$$

With the help of expressions (9) and (16), this modified Thouless criterion then yields the result

$$
\frac{m}{4 \pi a_{F}}+\frac{(2 m)^{3 / 2} \sqrt{\mu}}{4 \pi^{2}} \ln \left(\frac{8 \mu e^{\gamma} e^{1 / 3}}{\pi T_{c} e^{2}}\right)=0,
$$

as we had anticipated

In the BEC (strong-coupling) limit, on the other hand, the Popov bosoniclike self-energy acquires the form [17]

$$
\Sigma_{\text {Popov }}^{\mathrm{B}} \simeq-\frac{m k_{F}}{6 \pi^{2}}\left(k_{F} a_{F}\right)^{2} .
$$


In the following, the Popov correction [Eq. (13)] (with $Q=$ 0 ) is included numerically throughout the whole BCS-BEC crossover, where it will turn out to give an important contribution to the calculation of the critical temperature. In this context, recovering numerically the analytic BCS [Eq. (16)] and BEC [Eq. (19)] limiting values will serve as an important test for the accuracy of the calculations. The procedure to set up the numerical calculation of $\Sigma_{\mathrm{Popov}}^{\mathrm{B}}$ for a generic value of the coupling $\left(k_{F} a_{F}\right)^{-1}$ is described in Appendix A.

\section{GMB contribution}

Akin to the Popov correction discussed above, also the GMB correction can be interpreted in terms of a bosoniclike self-energy $\Sigma_{\mathrm{GMB}}^{\mathrm{B}}$ shown in Fig. 2(b), which dresses the bare pair propagator $\Gamma_{0}$. In this respect, our approach is similar to the original GMB treatment [2], where the singularities of the "vertex part" of the two-particle Green's function [26] were identified by approaching $T_{c}$ from the normal phase. This contrasts with the treatment of Ref. [6] (see also Ref. [27]), where an induced interaction was added to the bare interaction directly in the linearized gap equation to approach $T_{c}$ from the superfluid phase. In these references, however, only the BCS (weak-coupling) limit was considered.

The present treatment of the GMB correction differs from all previous treatments on the same subject. This is because we consider the GMB correction not only in the BCS limit but also throughout the BCS-BEC crossover. And, contrary to previous treatments of the GMB correction where the BCSBEC crossover was considered [13,14], we take into account the full dependence on wave vector and frequency of the pair propagators $\Gamma_{0}$ that appear in Fig. 2(b). This dependence, in fact, will prove to be an essential ingredient for an appropriate description of the physics at the basis of the GMB correction.

The analytic expression of the GMB bosoniclike self-energy $\Sigma_{\text {GMB }}^{\mathrm{B}}$ depicted in Fig. 2(b) reads

$$
\begin{aligned}
& \Sigma_{\mathrm{GMB}}^{\mathrm{B}}(Q) \\
& =\int \frac{d \mathbf{k}}{(2 \pi)^{3}} T \sum_{n} \int \frac{d \mathbf{p}}{(2 \pi)^{3}} T \sum_{v_{p}} \int \frac{d \mathbf{q}}{(2 \pi)^{3}} T \sum_{v_{q}} \\
& \quad \times G_{0}(p-k+Q) G_{0}(k-p) G_{0}(p+q-k+Q) G_{0}(k) \\
& \quad \times G_{0}(q-k+Q) G_{0}(k-q) \Gamma_{0}(p+Q) \Gamma_{0}(q+Q) .
\end{aligned}
$$

In the following, we again limit ourselves to consider the case $Q=0$, whereby we set $\Sigma_{\mathrm{GMB}}^{\mathrm{B}}=\Sigma_{\mathrm{GMB}}^{\mathrm{B}}(Q=0)$.

Expression (20), which contains six single-particle propagators $G_{0}$ and two pair propagators $\Gamma_{0}$ with one fermionic and two bosonic four-vector integrations, is considerably more involved than the Popov counterpart [Eq. (13)], so its numerical calculation for $Q=0$ will also prove quite more challenging. The numerical strategies to deal with this complicated calculation are outlined in Sec. III A and further discussed in more detail in Appendix A. In this context, we note that, for the needs of the numerical calculations, expression (20) is fully symmetric under the interchange $p \leftrightarrow q$. Different sets of four-wave-vector integration and summation variables $(k, p, q)$, however, are useful to obtain the BCS and BEC limits of expression (20), to be considered next.
In addition, we anticipate that in the numerical calculations reported in Sec. III the pair propagator $\Gamma_{0}$, which enters the Popov [Eq. (13)] and GMB [Eq. (20)] bosoniclike selfenergies as well as the fermionic self-energy [Eq. (7)] used in density equation (8), will everywhere be replaced by the pair propagator $\Gamma$ dressed by a constant shift according to Eq. (44) below. The presence of this shift (either $\Sigma_{\mathrm{Popov}}^{\mathrm{B}}$ or $\Sigma_{\mathrm{GMB}}^{\mathrm{B}}$, or both) is required to avoid unwanted divergences that would otherwise occur when $T \rightarrow T_{c}$.

\section{E. Disentanglement of pairing and screening in the BCS limit}

To obtain the BCS limiting value of the GMB bosoniclike self-energy [Eq. (20)], it would appear natural to approximate both pair propagators $\Gamma_{0}$ therein by their asymptotic expression $-4 \pi a_{F} / m$ valid in the BCS limit, in a similar way to what we did in Eq. (14) for the Popov bosoniclike self-energy. However, for the GMB case adopting tout court this approximation for $\Gamma_{0}$ would make the GMB self-energy [Eq. (20)] diverge, and some care should be accordingly exerted in this context. For these reasons, it is relevant to spell out in detail the sequence of (approximate) steps to obtain the GMB result for $T_{c}$ [2]. In this way, it will also be clear that these approximations cannot simply be extended to the whole BCS-BEC crossover, for which a more complete approach is instead required.

To deal with the BCS limit of the GMB self-energy [Eq. (20)], it is convenient to rename the four-wave-vector variables like in Fig. 3(a) (where, again, only the case $Q=$ 0 is considered). If one would retain the full four-vector dependence of the two pair propagators $\Gamma_{0}$ appearing in this diagram, there would be no problem in the convergence of the corresponding sums and integrals over the fermionic fourvectors $\left(k, k^{\prime}, k^{\prime \prime}\right)$, but no analytic result could be obtained in this way.

In the weak-coupling limit, the GMB result can be derived from the general expression (20) through the following steps:

(i) Begin by taking each $\Gamma_{0}$ of the form $-4 \pi a_{F} / m$, independent of wave vector and frequency. By doing this, a particle-hole bubble of the form

$$
\begin{aligned}
\chi_{\mathrm{ph}}\left(k+k^{\prime}\right)= & \int \frac{d \mathbf{k}^{\prime \prime}}{(2 \pi)^{3}} T \sum_{n^{\prime \prime}} G_{0}\left(\mathbf{k}^{\prime \prime}, \omega_{n^{\prime \prime}}\right) \\
& \times G_{0}\left(\mathbf{k}^{\prime \prime}-\mathbf{k}-\mathbf{k}^{\prime}, \omega_{n^{\prime \prime}}-\omega_{n}-\omega_{n^{\prime}}\right) \\
= & \int \frac{d \mathbf{k}^{\prime \prime}}{(2 \pi)^{3}} \frac{f\left(\xi_{\mathbf{k}+\mathbf{k}^{\prime}+\mathbf{k}^{\prime \prime}}\right)-f\left(\xi_{\mathbf{k}^{\prime \prime}}\right)}{\xi_{\mathbf{k}+\mathbf{k}^{\prime}+\mathbf{k}^{\prime \prime}}-\xi_{\mathbf{k}^{\prime \prime}}-i\left(\omega_{n}+\omega_{n^{\prime}}\right)}
\end{aligned}
$$

appears in the central part of the diagram. This bubble identifies the simplest process associated with screening in a Fermi gas [28].

(ii) In the particle-hole bubble [Eq. (21)], set $\omega_{n}+\omega_{n^{\prime}}=0$, take $\mathbf{k}$ and $\mathbf{k}^{\prime}$ on a Fermi sphere with radius $k_{\mu}$, and average over their relative angle. The result is

$$
\bar{\chi}_{\mathrm{ph}}=-N(\mu) \ln (4 e)^{1 / 3},
$$

where $N(\mu)=(2 m)^{3 / 2} \sqrt{\mu} /\left(4 \pi^{2}\right)$ is the single-particle density of states (per spin component) taken at the chemical potential. This step is justified by the presence of the sums over the fourvectors $k$ and $k^{\prime}$ in Fig. 3(a), whereby the factors $G_{0}(k) G_{0}(-k)$ 

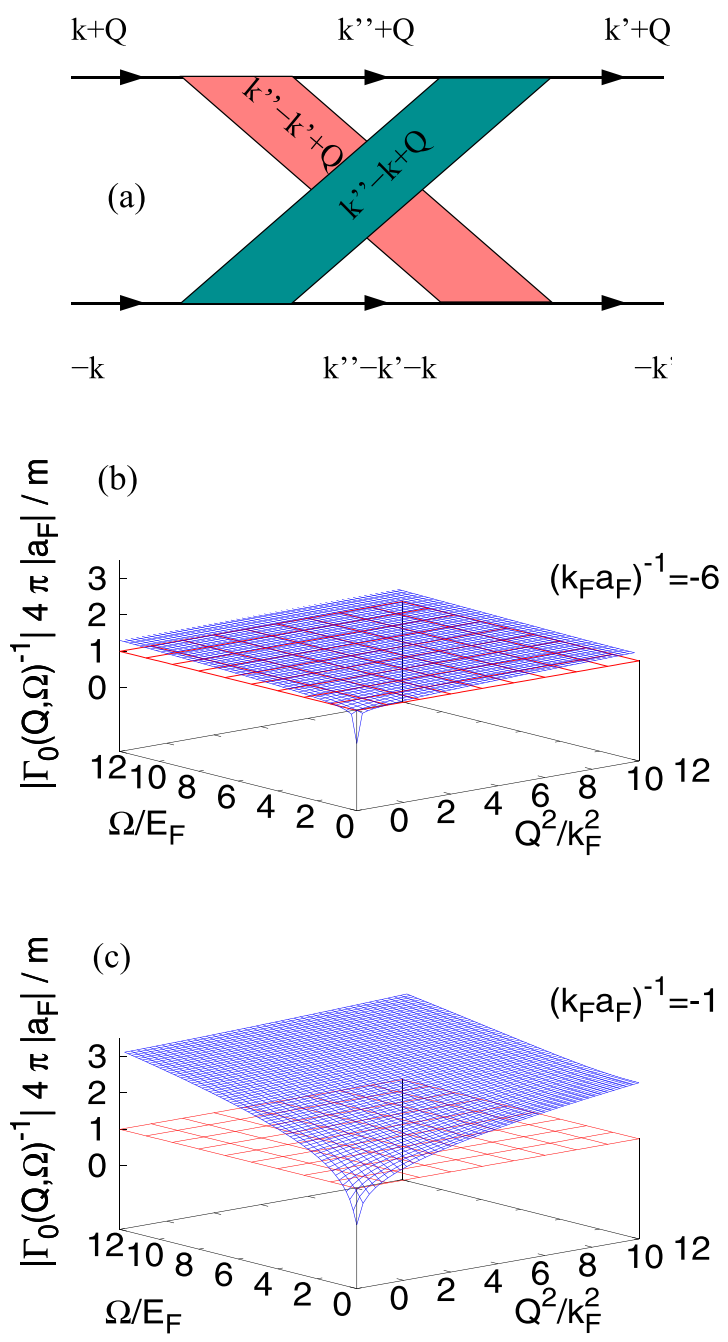

FIG. 3. (a) The GMB bosoniclike self-energy $\Sigma_{\text {GMB }}^{\mathrm{B}}$ of Fig. 2(b) is cast in a form suitable to obtain its BCS limit analytically. The magnitude of the inverse $\Gamma_{0}\left(\mathbf{Q}, \Omega_{v}\right)^{-1}$ of the pair propagator [in units of $\left.m /\left(4 \pi\left|a_{F}\right|\right)\right]$ is shown vs $\left(|\mathbf{Q}| / k_{F}\right)^{2}$ and $\Omega_{v} / E_{F}$ for the couplings (b) $\left(k_{F} a_{F}\right)^{-1}=-6$ in the extreme BCS limit and (c) $\left(k_{F} a_{F}\right)^{-1}=-1$ at the boundary between the BCS and the crossover regions.

and $G_{0}\left(k^{\prime}\right) G_{0}\left(-k^{\prime}\right)$ in the particle-particle bubbles on the leftand right-hand sides of the diagram are strongly peaked at $|\mathbf{k}|=k_{\mu}, \omega_{n}=0$ and $\left|\mathbf{k}^{\prime}\right|=k_{\mu}, \omega_{n^{\prime}}=0$.

(iii) In this way, the diagram of Fig. 3(a) is effectively disentangled into the product of three terms, namely, two particle-particle bubbles of the form (5) that appear on the left- and right-hand sides of the diagram and the (averaged) particle-hole bubble that appears in the central part of the diagram. Taken as they stand, the two particle-particle bubbles would diverge in the ultraviolet. However, owing to the convergence of the original diagram where the two pair propagators $\Gamma_{0}$ retain their full wave-vector and frequency dependence, one can safely make the integrals over $\mathbf{k}$ and $\mathbf{k}^{\prime}$ convergent again by replacing each particle-particle bubble [Eq. (5)] with its regularized version [Eq. (6)], whose integrand has the same behavior in the dominant region where $|\mathbf{k}| \simeq k_{\mu}, \omega_{n} \simeq 0$ and $\left|\mathbf{k}^{\prime}\right| \simeq k_{\mu}, \omega_{n^{\prime}} \simeq 0$. Accordingly, the GMB self-energy becomes

$$
\Sigma_{\mathrm{GMB}}^{\mathrm{B}}=\Sigma_{\mathrm{GMB}}^{\mathrm{B}}(Q=0) \simeq\left(-\frac{4 \pi a_{F}}{m}\right)^{2} R_{p p}(Q=0)^{2} \bar{\chi}_{\mathrm{ph}} .
$$

(Note in this context that the use here of the word "disentanglement" follows the original work of Feynman [29].)

(iv) The approximate result [Eq. (23)] affects the critical temperature by modifying the Thouless criterion, in the form

$$
\Gamma_{0}\left(Q=0 ; T_{c}, \mu_{c}\right)^{-1}-\Sigma_{\mathrm{GMB}}^{\mathrm{B}}(Q=0)=0
$$

in analogy with Eq. (17) for the Popov case. Making use of expression (4) for $\Gamma_{0}$ and of the result (23) for $\Sigma_{\mathrm{GMB}}^{\mathrm{B}}$, the generalized Thouless criterion [Eq. (24)] becomes

$$
\frac{m}{4 \pi a_{F}}+R_{p p}(Q=0)+\left(\frac{4 \pi a_{F}}{m} R_{p p}(Q=0)\right)^{2} \bar{\chi}_{\mathrm{ph}}=0 .
$$

(v) Expression (25) can be further simplified by noting that, for $T$ of the order of the BCS critical temperature, the Thouless criterion [Eq. (9)] is equivalent to writing $\frac{4 \pi a_{F}}{m} R_{p p}(Q=0)=$ -1 . Using this result, Eq. (25) reduces to its final form:

$$
\frac{m}{4 \pi a_{F}}+R_{p p}(Q=0)+\bar{\chi}_{\mathrm{ph}}=0 .
$$

With the result (22), one then obtains, in analogy with Eq. (18),

$$
\frac{m}{4 \pi a_{F}}+\frac{(2 m)^{3 / 2} \sqrt{\mu}}{4 \pi^{2}} \ln \left(\frac{8 \mu e^{\gamma}}{\pi T_{c} e^{2}(4 e)^{1 / 3}}\right)=0 .
$$

Upon setting $\mu=E_{F}$ in this expression, one gets that the value of the BCS critical temperature (2) is reduced by the factor $(4 e)^{1 / 3} \simeq 2.2$, as it was obtained in Ref. [2]. Otherwise, if the value [Eq. (12)] of $\mu$ within the non-self-consistent $t$-matrix approximation is adopted, the Popov contribution is also needed to get rid of the additional "spurious" factor $e^{-1 / 3}$ as discussed in Sec. IIC.

From the way it has been derived, it is clear that Eq. (26) holds only in the extreme BCS (weak-coupling) limit $\left(k_{F} a_{F}\right)^{-1} \ll-1$, whereby $\Gamma_{0}(Q)$ can be approximated by the constant term $-4 \pi a_{F} / m$. We have explicitly verified the validity of this approximation numerically, by plotting the magnitude of the inverse $\Gamma_{0}\left(\mathbf{Q}, \Omega_{v}\right)^{-1}$ of the pair propagator in units of $m /\left(4 \pi\left|a_{F}\right|\right)$, in Fig. 3(b) for the coupling values $\left(k_{F} a_{F}\right)^{-1}=-6$ in the extreme BCS (weak-coupling) limit and in Fig. 3(c) for the coupling value $\left(k_{F} a_{F}\right)^{-1}=-1$ at the boundary between the BCS and the crossover regions. From these plots, one indeed verifies that in the extreme BCS (weak-coupling) limit to a good approximation $\Gamma_{0}\left(\mathbf{Q}, \Omega_{v}\right)$ can be considered constant over a large plateau in the $\left(|\mathbf{Q}|, \Omega_{v}\right)$ plane. This property, however, no longer holds already at the boundary between the BCS and the crossover regions, where strong deviations of $\Gamma_{0}$ from constancy appear evident. In spite of this property, in Refs. [13,14] the critical temperature was calculated by relying on the result in Eq. (26) not only in the BCS limit but also across the whole BCS-BEC crossover.

In the following, we remedy this shortcoming by maintaining the full $\mathbf{Q}$ and $\Omega_{v}$ dependence of $\Gamma_{0}$ in expression (20) of the GMB self-energy $\Sigma_{\mathrm{GMB}}^{\mathrm{B}}$. The corresponding numerical calculation are reported in Sec. III throughout the BCS-BEC 


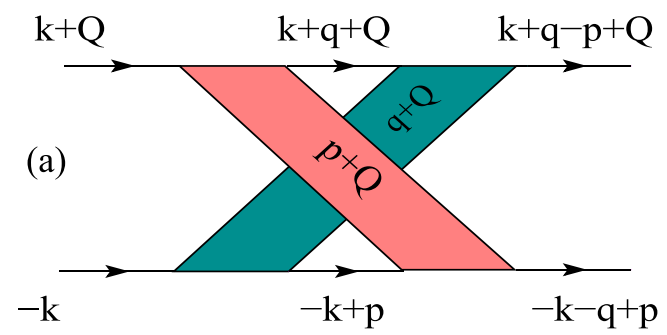

(b)

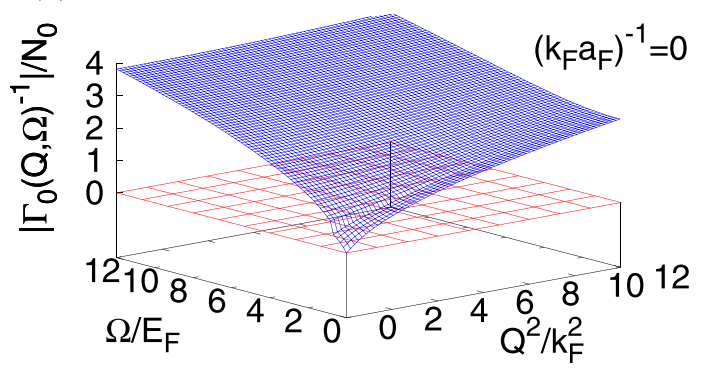

(c)

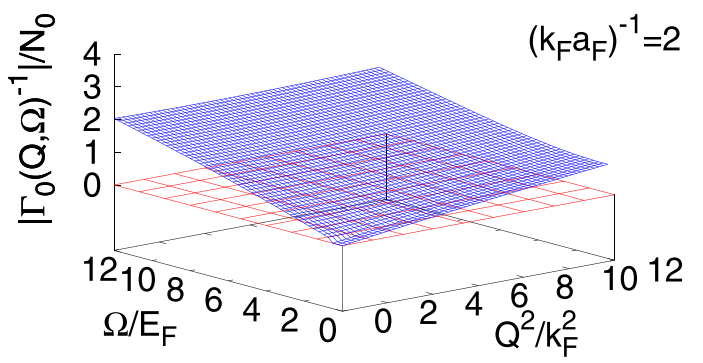

FIG. 4. (a) The GMB bosoniclike self-energy $\Sigma_{\text {GMB }}^{\mathrm{B}}$ of Fig. 2(b) is cast in a form suitable to obtain its BEC limit analytically. The magnitude of the inverse $\Gamma_{0}\left(\mathbf{Q}, \Omega_{v}\right)^{-1}$ of the pair propagator [in units of $\left.N_{0}=N\left(\mu=E_{F}\right)\right]$ is shown vs $\left(|\mathbf{Q}| / k_{F}\right)^{2}$ and $\Omega_{v} / E_{F}$ for the couplings (b) $\left(k_{F} a_{F}\right)^{-1}=0$ at unitarity and (c) $\left(k_{F} a_{F}\right)^{-1}=2$ in the BEC regime.

crossover. In particular, we anticipate that our calculation will be able to reproduce in a totally numerical fashion the GMB result for the reduction of the value of the critical temperature [Eq. (2)] by the factor $(4 e)^{1 / 3} \simeq 2.2$ in the extreme weakcoupling limit, thus confirming the validity of the nontrivial weak-coupling approximations leading to the GMB result.

\section{F. Transmuting of screening into pairing in the BEC limit}

To deal with the BEC (strong-coupling) limit of the GMB bosoniclike self-energy [Eq. (20)], it is convenient to rename the four-wave-vector variables like in Fig. 4(a) (where the case $Q=0$ is again considered). In this limit, we are going to show analytically that keeping the full $\mathbf{Q}$ and $\Omega_{\nu}$ dependence in the pair propagators $\Gamma_{0}$ of the GMB contribution is essential for a correct evaluation of this quantity. This need can be anticipated by looking at the much stronger $\mathbf{Q}$ and $\Omega_{v}$ dependence of $\left|\Gamma_{0}\left(\mathbf{Q}, \Omega_{\nu}\right)^{-1}\right|$ that occurs in the BEC with respect to the BCS limit, as shown in Fig. 4(c). For comparison, in Fig. 4(b) the shape of $\left|\Gamma_{0}\left(\mathbf{Q}, \Omega_{v}\right)^{-1}\right|$ is also shown at unitarity. [Note the change of normalization for $\left|\Gamma_{0}\left(\mathbf{Q}, \Omega_{v}\right)^{-1}\right|$ in Figs. 4(b) and 4(c) with respect to Figs. 3(b) and 3(c).]

In the BEC limit $\mu / T \rightarrow-\infty$ we are interested in, the pair propagators entering the diagram of Fig. 4(a) have the approximate form [30]

$$
\Gamma_{0}\left(\mathbf{q}, \Omega_{q}\right)=\frac{D\left(\mathbf{q}, \Omega_{q}\right)}{i \Omega_{q}-\xi_{\mathbf{q}}^{B}},
$$

where

$$
D\left(\mathbf{q}, \Omega_{q}\right)=-\frac{4 \pi}{m^{2} a_{F}}\left(1+\sqrt{1+\frac{\xi_{\mathbf{q}}^{B}-i \Omega_{q}}{\epsilon_{0}}}\right) .
$$

In these expressions, $\epsilon_{0}=\left(m a_{F}^{2}\right)^{-1}$ is the binding energy of the two-fermion problem in vacuum and $\xi_{\mathbf{q}}^{B}=\mathbf{q}^{2} /(4 m)-$ $\mu_{B}$, where $\mu_{B}=2 \mu+\epsilon_{0}$ is the chemical potential for the composite bosons that form in this limit. When extended to the complex frequency plane by letting $i \Omega_{q} \rightarrow z$ in Eqs. (28) and (29), the function $\Gamma_{0}(\mathbf{q}, z)$ has a pole at $z=\xi_{\mathbf{q}}^{B}$ and a cut along the real frequency axis for $z \geqslant \xi_{\mathbf{q}}^{B}+\epsilon_{0}$.

The integrations occurring in the expression of the GMB bosoniclike self-energy $\Sigma_{\mathrm{GMB}}^{B}$ [cf. the diagram of Fig. 4(a)] can be done by first considering the sums over the bosonic Matsubara frequencies $\Omega_{p}$ and $\Omega_{q}$. These sums can both be transformed into contour integrals in the complex $z$ plane by using the bosonic distribution $b(z)=\left(\exp \left\{z /\left(k_{B} T\right)\right\}-1\right)^{-1}$ [31]. Let $C$ be a contour in this plane which encircles poles and branch cut clockwise. Owing to the presence of the function $b(z)$, the contribution from the cuts of $\Gamma_{0}(p)$ and $\Gamma_{0}(q)$ are exponentially suppressed in the BEC limit (when $\epsilon_{0}$ is the largest energy scale) and need not be considered. Similarly, the contribution from the poles of $G_{0}(k+q)$ and $G_{0}(-k+p)$ where $p$ and $q$ appear with a positive sign are also exponentially suppressed. Therefore, only the poles of $\Gamma_{0}(p)$ and $\Gamma_{0}(q)$ and of $G_{0}(k+q-p)$ and $G_{0}(-k-q+p)$ (where $p$ or $q$ appear with a negative sign) give a finite contribution.

Specifically, three contributions result by doing the sums over $\Omega_{q}$ and $\Omega_{p}$ in the sequence, which arise respectively from the poles of (i) $\Gamma_{0}(q)$ and $\Gamma_{0}(p)$, (ii) $\Gamma_{0}(q)$ and $G_{0}(k+q-p)$, and (iii) $\Gamma_{0}(p)$ and $G_{0}(-k-q+p)$.

Contribution (i) yields

$$
\begin{aligned}
\Sigma_{\mathrm{GMB}}^{B(\mathrm{i})} \cong & \frac{8 \pi}{m^{2} a_{F}} \int \frac{d \mathbf{q}}{(2 \pi)^{3}} b\left(\xi_{\mathbf{q}}^{B}\right) \\
& \times \frac{8 \pi}{m^{2} a_{F}} \int \frac{d \mathbf{p}}{(2 \pi)^{3}} b\left(\xi_{\mathbf{p}}^{B}\right) \int d k G_{0}(k)^{3} G_{0}(-k)^{3},
\end{aligned}
$$

where

$$
\int d k G_{0}(k)^{3} G_{0}(-k)^{3} \cong \frac{15 m^{5} a_{F}^{7}}{256 \pi}
$$

with the shorthand notation

$$
\int d k=\int \frac{d \mathbf{k}}{(2 \pi)^{3}} T \sum_{\omega_{k}}
$$

and similarly for the other integrals. Note that this contribution (which is of second order in the bosonic density $n_{B}=n / 2$ ) could have been obtained by neglecting the $p$ and $q$ dependence 
everywhere in the $G_{0}$ of the diagram in Fig. 4(a) and by approximating further:

$$
\begin{aligned}
\int d q \Gamma_{0}(q) & \cong-\frac{8 \pi}{m^{2} a_{F}} \int \frac{d \mathbf{q}}{(2 \pi)^{3}} T \sum_{\Omega_{q}} \frac{e^{i \Omega_{q} \eta}}{i \Omega_{q}-\xi_{\mathbf{q}}^{B}} \\
& =\frac{8 \pi}{m^{2} a_{F}} \int \frac{d \mathbf{q}}{(2 \pi)^{3}} b\left(\xi_{\mathbf{q}}^{B}\right)=\frac{8 \pi}{m^{2} a_{F}} n_{B},
\end{aligned}
$$

where $\eta$ is a positive infinitesimal.

Contribution (ii) yields instead

$$
\Sigma_{\mathrm{GMB}}^{B(\mathrm{ii})} \simeq \frac{8 \pi}{m^{2} a_{F}} \int \frac{d \mathbf{q}}{(2 \pi)^{3}} b\left(\xi_{\mathbf{q}}^{B}\right) \int \frac{d \mathbf{p}}{(2 \pi)^{3}} \phi(\mathbf{p}),
$$

where the sum over the fermionic frequency $\omega_{k}$ has been performed analytically and with the definition

$$
\phi(\mathbf{p})=\int \frac{d \mathbf{k}}{(2 \pi)^{3}} \frac{\Gamma_{0}\left(\mathbf{p},-\xi_{\mathbf{k}}-\xi_{\mathbf{k}-\mathbf{p}}\right)}{\left(2 \xi_{\mathbf{k}}\right)^{2}\left(2 \xi_{\mathbf{k}-\mathbf{p}}\right)^{2}} .
$$

Here, $\Gamma_{0}\left(\mathbf{p},-\xi_{\mathbf{k}}-\xi_{\mathbf{k}-\mathbf{p}}\right)$ is obtained from $\Gamma_{0}\left(\mathbf{p}, \Omega_{p}\right)$ given by Eqs. (28) and (29) with the replacement $i \Omega_{p} \rightarrow-\xi_{\mathbf{k}}-$ $\xi_{\mathbf{k}-\mathbf{p}}$. Note that, the numerator (29) of the expression (28) contributes to the integration in Eq. (35). It is for this reason that, contrary to the expression (30), only one power of the bosonic density $n_{B}$ appears eventually in the expression (34). An expression identical to (34) results also from the contribution (iii) (apart from the interchange of the wave vectors $\mathbf{q}$ and $\mathbf{p})$.

There remains to calculate the function $\phi(\mathbf{p})$ given by Eq. (35). To this end, it is convenient to change the integration variable into $\mathbf{k}^{\prime}=\mathbf{k}-\mathbf{p} / 2$, rescale the magnitude of the wave vectors by $\tilde{k}^{\prime}=\left|\mathbf{k}^{\prime}\right| a_{F}$ and $\tilde{p}=|\mathbf{p}| a_{F}$, and introduce the notation

$$
A\left(\tilde{k}^{\prime}, \tilde{p}\right)=\frac{1+\tilde{k}^{\prime 2}+\tilde{p}^{2} / 4}{\tilde{k}^{\prime} \tilde{p}} .
$$

In this way, the integral over the angle between $\mathbf{k}^{\prime}$ and $\mathbf{p}$ can be done analytically, yielding

$$
\phi(\mathbf{p})=\frac{2 m^{3} a_{F}^{4}}{\pi} \frac{1}{\mathbf{p}^{2}} F(\tilde{p}),
$$

where

$$
\begin{aligned}
F(\tilde{p})= & \int_{0}^{\infty} d \tilde{k} \frac{\left(1+\sqrt{2+\tilde{k}^{2}+\tilde{p}^{2} / 2}\right)}{\left(2+2 \tilde{k}^{2}+\tilde{p}^{2}\right)\left(1+\tilde{k}^{2}+\tilde{p}^{2} / 4\right)^{2}} \\
& \times\left[\frac{1}{A(\tilde{k}, \tilde{p})^{2}-1}+\frac{1}{A(\tilde{k}, \tilde{p})} \operatorname{arctanh}\left(\frac{1}{A(\tilde{k}, \tilde{p})}\right)\right] .
\end{aligned}
$$

To obtain this expression, we have neglected the small energy scale $\mu_{B}$ with respect to $\epsilon_{0}$. We can thus rewrite in Eq. (34)

$$
\int \frac{d \mathbf{p}}{(2 \pi)^{3}} \phi(\mathbf{p})=\frac{m^{3} a_{F}^{3}}{\pi^{3}} \int_{0}^{\infty} d \tilde{p} F(\tilde{p}),
$$

where a numerical calculation gives the value $\tilde{I}=0.25974$ for the integral of $F(\tilde{p})$ in Eq. (39).
In conclusion, the sum of the above three contributions (i)(iii) can be written in the compact form

$$
\begin{aligned}
\Sigma_{\mathrm{GMB}}^{B} \simeq & \int \frac{d \mathbf{p}}{(2 \pi)^{3}} \int \frac{d \mathbf{q}}{(2 \pi)^{3}} \\
& \times\left\{\frac{8 \pi}{m^{2} a_{F}}\left[b\left(\xi_{\mathbf{q}}^{B}\right) \phi(\mathbf{p})+b\left(\xi_{\mathbf{p}}^{B}\right) \phi(\mathbf{q})\right]\right. \\
& \left.+\left(\frac{8 \pi}{m^{2} a_{F}}\right)^{2} \frac{15 m^{5} a_{F}^{7}}{256 \pi} b\left(\xi_{\mathbf{p}}^{B}\right) b\left(\xi_{\mathbf{q}}^{B}\right)\right\} \\
\simeq & \frac{16 \tilde{I}}{\pi^{2}} \frac{m k_{F}}{6 \pi^{2}}\left(k_{F} a_{F}\right)^{2}
\end{aligned}
$$

to the leading order in the small parameter $k_{F} a_{F}$. Apart from a sign, this result differs from the corresponding Popov result [Eq. (19)] by the constant factor $16 \tilde{I} / \pi^{2} \simeq 0.421$.

Note that the power-law dependence of expression (40) on the small parameter $\left(k_{F} a_{F}\right) \ll 1$ contrasts with the exponential dependence of the particle-hole bubble [Eq. (21)] on the (square of the) coupling parameter $\left(k_{F} a_{F}\right)^{-1}$, which would be obtained by extending expression (21) to the BEC limit $\left(k_{F} a_{F}\right)^{-1} \rightarrow+\infty$. In fact, no trace of the particle-hole bubble [Eq. (21)] appears in the derivation of the result in Eq. (40), where only particle-particle processes, which are relevant to the scattering between composite bosons, correctly occur in the BEC limit. This result confirms that screening processes show up in the GMB contribution only in the opposite BCS limit $\left(k_{F} a_{F}\right)^{-1} \rightarrow-\infty$ treated originally by Gorkov and MelikBarkhudarov [2] and discussed in Sec. IIE.

Result (40) is further considered in Appendix B, where the GMB self-energy $\Sigma_{\mathrm{GMB}}^{B}$ is shown to contribute to the scattering length $a_{B}$ of composite bosons that form in the BEC limit. In the following section, result (40) instead represents a benchmark for the numerical calculation in the (extreme) BEC limit.

\section{NUMERICAL RESULTS}

In this section, we implement numerically the inclusion of the Popov and GMB corrections into the non-self-consistent $t$-matrix approximation. We are specifically concerned with determining how these corrections affect the value of the critical temperature $T_{c}$ throughout the BCS-BEC crossover. In the process, the numerical accuracy of the calculation of the Popov and GMB corrections is tested against the analytic results obtained in Sec. II in the BCS and BEC limits. Our numerical results for $T_{c}$ are also compared with quantum Monte Carlo calculations which are available in an extended region of coupling about unitarity and with the experimental data which are available at unitarity.

\section{A. Numerical strategies at $\boldsymbol{T}_{c}$}

In the Popov [ $\Sigma_{\text {Popov }}^{B}$ of Eq. (13)] and GMB $\left[\Sigma_{\mathrm{GMB}}^{B}\right.$ of Eq. (20)] bosoniclike self-energies depicted in Fig. 2, all pair propagators were taken to be bare ones. This choice was sufficient for obtaining the analytic results in the BCS and BEC limit. When implementing the numerical calculations for these bosoniclike self-energies, however, care must be exerted based on the fact that the bare pair propagator $\Gamma_{0}(Q)$ becomes singular at $Q=0$ for specific values of $T_{c}$ and $\mu_{c}$ according 
to the Thouless criterion [Eq. (9)]. This problem is definitely bound to show up numerically in the BCS limit, where one knows that the GMB correction decreases the value of the critical temperature by a factor of 2.2 with respect to the BCS result.

To overcome this problem, it is clear that some degree of self-consistency must unavoidably be included in the calculation. However, to keep at the same time the calculation for determining $T_{c}$ as simple as possible (thus avoiding increasing its complexity beyond affordable limits), self-consistency is implemented in practice according to the following scheme.

We begin by considering Eq. (4), in the form

$$
\Gamma_{0}(Q ; T, \mu)^{-1}=-\frac{m}{4 \pi a_{F}}-R_{\mathrm{pp}}(Q ; T, \mu),
$$

where $R_{\mathrm{pp}}$ is given by expression (6) and the dependence on the thermodynamic quantities $(T, \mu)$ has been explicitly indicated. By normalizing $\Gamma_{0}^{-1}$ in terms of the single-particle density of states (per spin component) at the Fermi level, $N_{0}=N\left(\mu=E_{F}\right)=m k_{F} /\left(2 \pi^{2}\right)$, the coupling $\left(k_{F} a_{F}\right)^{-1}$ is seen to appear explicitly only in the first term on the right-hand side of Eq. (41). In particular, for given value of $\left(k_{F} a_{F}\right)^{-1}$ the condition $\Gamma_{0}\left(Q=0 ; T_{c}, \mu_{c}\right)^{-1}=0$ determines a set of values $\left\{T_{c}, \mu_{c}\right\}$ consistently with the equation

$$
\frac{m}{4 \pi a_{F}}+R_{\mathrm{pp}}\left(Q=0 ; T_{c}, \mu_{c}\right)=0 .
$$

For each of these pairs of values, we can then rewrite Eq. (41) in the form

$$
\Gamma_{0}\left(Q ; T_{c}, \mu_{c}\right)^{-1}=-R_{\mathrm{pp}}\left(Q ; T_{c}, \mu_{c}\right)+R_{\mathrm{pp}}\left(Q=0 ; T_{c}, \mu_{c}\right) .
$$

Upon entering expression (43) for $\Gamma_{0}(Q)$ into the fermionic self-energy [Eq. (7)] and then into density equation (8), in order to determine uniquely a pair of values $T_{c}$ and $\mu_{c}$ for given $\left(k_{F} a_{F}\right)^{-1}$, it turns out that the density equation does not depend explicitly on the coupling $\left(k_{F} a_{F}\right)^{-1}$ but only on the pair $\left(T_{c}, \mu_{c}\right)$.

Next, we consider the effect of the bosoniclike self-energy $\Sigma^{B}$ (either $\Sigma_{\text {Popov }}^{B}$ or $\Sigma_{\mathrm{GMB}}^{B}$, or both) calculated at $Q=0$ only, by introducing the dressed pair propagator:

$$
\Gamma(Q ; T, \mu)^{-1}=\Gamma_{0}(Q ; T, \mu)^{-1}-\Sigma^{B}(T, \mu) .
$$

In this case, a new set of values $\left\{\bar{T}_{c}, \bar{\mu}_{c}\right\}$ is determined by the generalized Thouless criterion

$$
\begin{aligned}
\Gamma\left(Q=0 ; \bar{T}_{c}, \bar{\mu}_{c}\right)^{-1}= & \Gamma_{0}\left(Q=0 ; \bar{T}_{c}, \bar{\mu}_{c}\right)^{-1} \\
& -\Sigma^{B}\left(\bar{T}_{c}, \bar{\mu}_{c}\right)=0 .
\end{aligned}
$$

In this way, Eq. (44) becomes, for given value of $\left(\bar{T}_{c}, \bar{\mu}_{c}\right)$ and a generic value of $Q$,

$$
\begin{aligned}
\Gamma\left(Q ; \bar{T}_{c}, \bar{\mu}_{c}\right)^{-1} & =\Gamma_{0}\left(Q ; \bar{T}_{c}, \bar{\mu}_{c}\right)^{-1}-\Sigma^{B}\left(\bar{T}_{c}, \bar{\mu}_{c}\right) \\
& =\Gamma_{0}\left(Q ; \bar{T}_{c}, \bar{\mu}_{c}\right)^{-1}-\Gamma_{0}\left(Q=0 ; \bar{T}_{c}, \bar{\mu}_{c}\right)^{-1} \\
& =-R_{\mathrm{pp}}\left(Q ; \bar{T}_{c}, \bar{\mu}_{c}\right)+R_{\mathrm{pp}}\left(Q=0 ; \bar{T}_{c}, \bar{\mu}_{c}\right),
\end{aligned}
$$

where in the last line Eq. (41) has been used. Note that the right-hand sides of Eqs. (43) and (46) are formally identical to each other, apart from the different set of values $\left(T_{c}, \mu_{c}\right)$ and $\left(\bar{T}_{c}, \bar{\mu}_{c}\right)$ on which they depend. This remark implies that the replacement $\Gamma_{0}(Q)^{-1} \rightarrow \Gamma(Q)^{-1}$ in the expressions of the Popov self-energy [Eq. (13)] and the GMB self-energy [Eq. (20)] amounts to considering a new set of values $\left(\bar{T}_{c}, \bar{\mu}_{c}\right)$ in the place of the old ones $\left(T_{c}, \mu_{c}\right)$. This is also true when this replacement is made in the density equation [Eq. (8)], which in this way depends only on the pairs $\left(\bar{T}_{c}, \bar{\mu}_{c}\right)$ but not explicitly on the coupling $\left(k_{F} a_{F}\right)^{-1}$. The new values $\left(\bar{T}_{c}, \bar{\mu}_{c}\right)$ are then determined by the generalized Thouless criterion (45), in the form

$$
\begin{aligned}
& -\Gamma_{0}\left(Q=0 ; \bar{T}_{c}, \bar{\mu}_{c}\right)^{-1}+\Sigma^{B}\left(\bar{T}_{c}, \bar{\mu}_{c}\right) \\
& \quad=\frac{m}{4 \pi a_{F}}+R_{\mathrm{pp}}\left(Q=0 ; \bar{T}_{c}, \bar{\mu}_{c}\right)+\Sigma^{B}\left(\bar{T}_{c}, \bar{\mu}_{c}\right)=0
\end{aligned}
$$

for a given coupling $\left(k_{F} a_{F}\right)^{-1}$. Note that Eq. (47) has the same structure of Eq. (24), although with a more general expression for the bosoniclike self-energy $\Sigma^{B}$.

We again emphasize that the above simplified procedure, for including some degree of self-consistency in the pair propagator $\Gamma_{0}$, relies on the fact that we are considering only the $Q=0$ value of $\Sigma^{B}(Q)$ and limit ourselves to determine the critical temperature $T_{c}$. In addition, we note that, owing to the formal analogy between Eqs. (43) and (46), the density equation (8) (with the dressed $\Gamma$ in the place of the bare $\Gamma_{0}$ ) is formally identical to its counterpart within the (non-selfconsistent) $t$-matrix approximation. As a consequence, the solution of the generalized Thouless criterion [Eq. (47)] plus the associated density equation to get a new pair of values $\left(\bar{T}_{c}, \bar{\mu}_{c}\right)$ amounts in practice to fixing an old pair of values $\left(T_{c}, \mu_{c}\right)$ that satisfy the original Thouless criterion [Eq. (42)] plus the corresponding density equation for given coupling $g=\left(k_{F} a_{F}\right)^{-1}$, and then finding the value of the modified coupling $\bar{g}$ for which Eq. (47) is satisfied.

Finally, we comment that more sophisticated degrees of self-consistency with respect to the one adopted here (like the replacement of the bare fermionic single-particle propagator $G_{0}$ by the dressed one $G$, everywhere $G_{0}$ appears in the relevant diagrams) are bound to result in an exceedingly difficult numerical calculation, especially as far as the GMB self-energy $\Sigma_{\mathrm{GMB}}^{B}$ is concerned. This need to restrict to the $G_{0}$ will be apparent in the discussion of Appendix A, where the calculation of the Popov and GMB diagrams is implemented in detail.

\section{B. Bosoniclike self-energies and generalized Thouless criterion}

To solve the generalized Thouless criterion [Eq. (47)], knowledge is required of the bosoniclike self-energy (either Popov or GMB, or both), calculated at the self-consistent values $\left(\bar{T}_{c}, \bar{\mu}_{c}\right)$ for a given value of the coupling $\left(k_{F} a_{F}\right)^{-1}$.

A plot of $\Sigma_{\text {Popov }}^{\mathrm{B}}$ and $\Sigma_{\text {GMB }}^{\mathrm{B}}$ is shown in Fig. 5(a) throughout the BCS-BEC crossover, with both quantities calculated at the values of $\left(\bar{T}_{c}, \bar{\mu}_{c}\right)$ of the full theory. Note that these two quantities have somewhat opposite behavior, with a comparable magnitude across the whole crossover. The limiting behaviors of both quantities in the (extreme) BCS limit are shown in Fig. 5(b). In particular, the limiting BCS values for $\left(k_{F} a_{F}\right) \rightarrow$ $0^{-}$have been obtained in both cases by fitting the numerical results with a quadratic polynomial and then extrapolating the curve to $k_{F} a_{F}=0$, recovering in this way with very good 


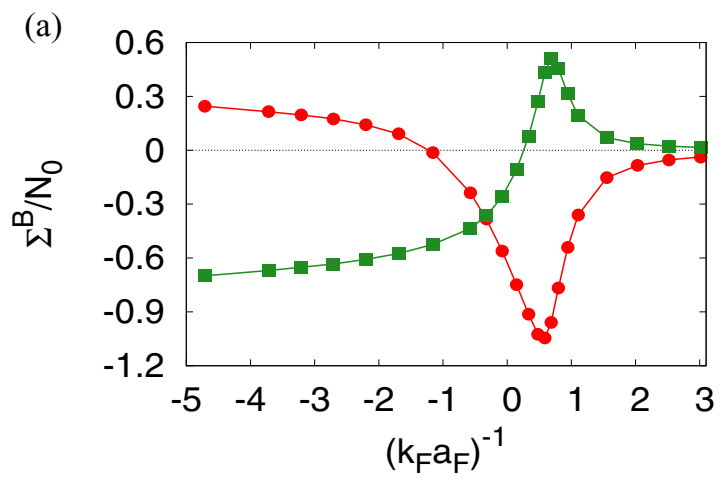

(b)

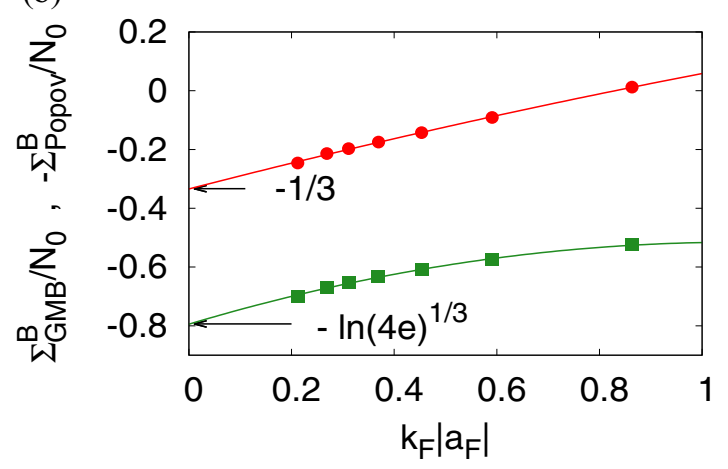

(c)

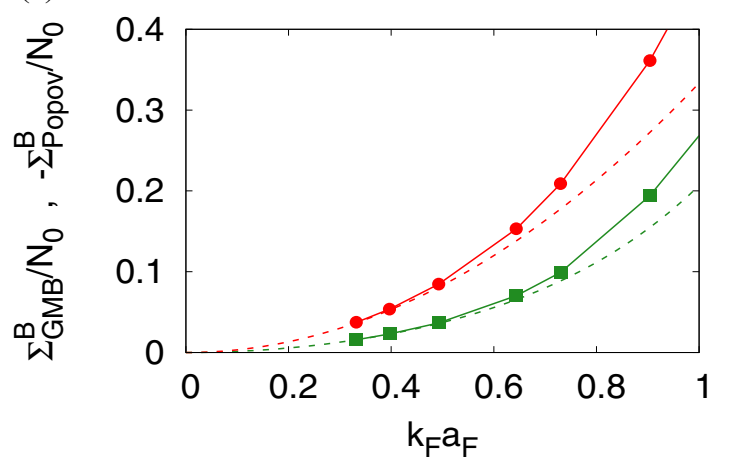

FIG. 5. (a) Bosoniclike self-energies $\Sigma_{\text {Popov }}^{\mathrm{B}}$ (circles) and $\Sigma_{\mathrm{GMB}}^{\mathrm{B}}$ (squares) (in units of the single-particle density of states $N_{0}$ ) vs the coupling $\left(k_{F} a_{F}\right)^{-1}$. Both quantities are calculated with the values of $\left(\bar{T}_{c}, \bar{\mu}_{c}\right)$ of the full theory. (b) $\Sigma_{\text {Popov }}^{\mathrm{B}}$ and $\Sigma_{\mathrm{GMB}}^{\mathrm{B}}$ vs $k_{F}\left|a_{F}\right|$ (with $\left.a_{F}<0\right)$ obtained numerically in the interval $(0,1)$. The limiting values for $k_{F}\left|a_{F}\right| \rightarrow 0$ are shown to recover the results (16) and (22), respectively, through an extrapolation procedure represented in each case by a solid line. (c) $\Sigma_{\text {Popov }}^{\mathrm{B}}$ and $\Sigma_{\mathrm{GMB}}^{\mathrm{B}}$ vs $k_{F} a_{F}$ (with $a_{F}>0$ ) obtained numerically (symbols plus solid lines) in the interval $(0,1)$ are compared, respectively, with the analytic behaviors (19) and (40) (dashed lines). [Note that in (b) and (c) $\Sigma_{\text {Popov }}^{\mathrm{B}}$ is multiplied by a minus sign.]

accuracy the Popov value of 1/3 given by Eq. (16) and the GMB value of $-\ln (4 e)^{1 / 3}$ given by Eq. (22) [in units of $N(\mu)$ with $\mu=E_{F}$ ]. Figure 5(c) shows further the limiting behaviors of the Popov and GMB bosoniclike self-energies in the BEC limit for small values of $k_{F} a_{F}$. In this case, our numerical calculations are compared with the analytic expressions for the Popov [Eq. (19)] and GMB [Eq. (40)] contributions. These

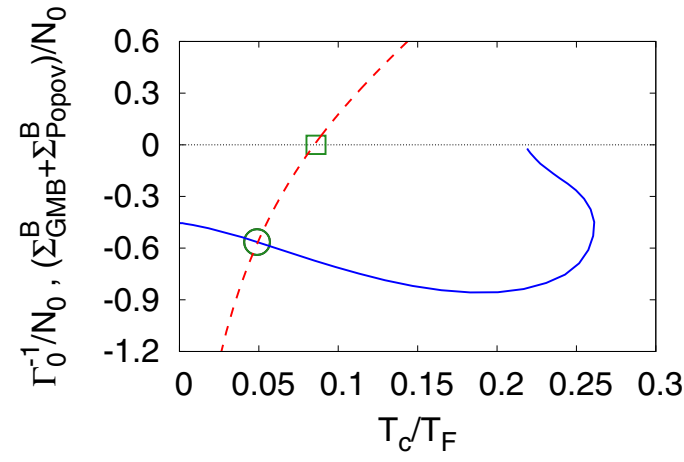

FIG. 6. The graphical procedure for determining numerically the intersection between $\Gamma_{0}^{-1}(Q=0)$ (dashed line) and $\Sigma_{\mathrm{GMB}}^{\mathrm{B}}+\Sigma_{\text {Popov }}^{\mathrm{B}}$ (solid line) is shown for the coupling $\left(k_{F} a_{F}\right)^{-1}=-1.0$ (both quantities are in units of single-particle density of states, $N_{0}$ ). Here, $T_{c}^{t \text {-matrix }}=0.08485 T_{F}$ corresponds to the intersection given by the square and $T_{c}^{\text {Popov+GMB }}=0.04860 T_{F}$ corresponds to the intersection given by the circle.

limiting analytic behaviors are quite well reproduced by our numerical calculation, thus providing a stringent test on its accuracy.

Figure 6 reports an example about the way the generalized Thouless criterion [Eq. (47)] is solved in practice for given coupling. It amounts to finding the intersection between the curves of $\Gamma_{0}^{-1}(Q=0)$ and $\Sigma_{\mathrm{GMB}}^{\mathrm{B}}+\Sigma_{\text {Popov }}^{\mathrm{B}}$ vs $T / T_{F}$. One begins by drawing the function $\Gamma_{0}^{-1}\left(Q=0 ; T_{c}, \mu_{c}\right)$ vs $T_{c} / T_{F}$ (dashed line), where the value of $T_{c}$ and of the associated $\mu_{c}$ are consistent with density equation (8). Since this function given by Eq. (41) depends explicitly on the coupling, the plot reported in Fig. 6 corresponds to a specific value of $\left(k_{F} a_{F}\right)^{-1}$. The value of $T_{c}$ at which $\Gamma_{0}^{-1}$ crosses zero (identified by the square in Fig. 6) then corresponds to the (non-self-consistent) $t$-matrix approximation (cf. the discussion of Sec. III A. Next one draws the bosoniclike self-energy $\Sigma_{\mathrm{GMB}}^{\mathrm{B}}+\Sigma_{\text {Popov }}^{\mathrm{B}}$ vs $T_{c} / T_{F}$ (solid line), which also depends on the pair $\left(T_{c}, \mu_{c}\right)$ as specified above. The intersection of this curve with the function $\Gamma_{0}^{-1}$ (identified by the circle in Fig. 6) provides eventually the value of $T_{c}$ (and thus also of $\mu_{c}$ ) with the Popov and GMB corrections included for the given coupling.

\section{Critical temperature}

The complete dependence of the critical temperature $T_{c}$ on coupling obtained in this way is reported in Fig. 7(a). This figure shows the results obtained by several approximations: (i) the most complete result, $T_{c}^{(\mathrm{GMB}+\text { Popov) }}$, obtained by including both $\Sigma_{\mathrm{GMB}}^{\mathrm{B}}$ and $\Sigma_{\text {Popov }}^{\mathrm{B}}$ (solid line); (ii) the partial result, $T_{c}^{(\mathrm{GMB})}$, obtained by including $\Sigma_{\mathrm{GMB}}^{\mathrm{B}}$ only (dashed line); (iii) the partial result, $T_{c}^{\text {(Popov) }}$, obtained by including $\Sigma_{\text {Popov }}^{\mathrm{B}}$ only (dotted line); (iv) $T_{c}^{(t \text {-matrix) }}$ corresponding to the (nonself-consistent) $t$-matrix approximation (dash-dotted line); and (v) the BCS result, $\bar{T}_{c}^{(\mathrm{BCS})}$, obtained at the mean-field level (i.e., with no inclusion of pairing fluctuations) throughout the BCS-BEC crossover [32] (dashed double-dotted line), which extends expression (2) away from the extreme weak coupling.

From this figure it appears that curves (i)-(iii) are obtained from curve (iv) by a nonuniform "stretching" of the coupling 
(a)

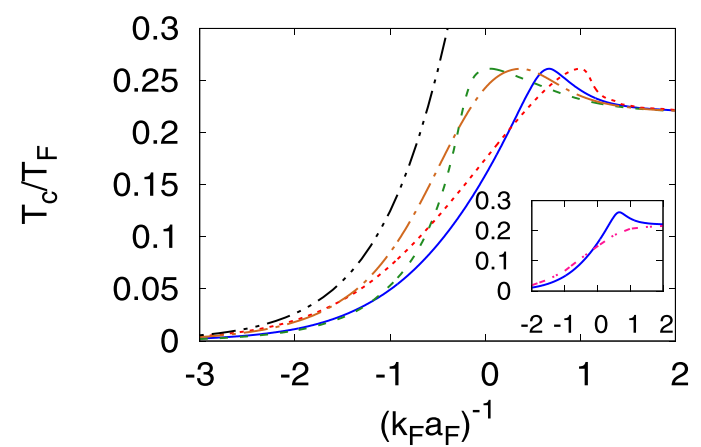

(b)

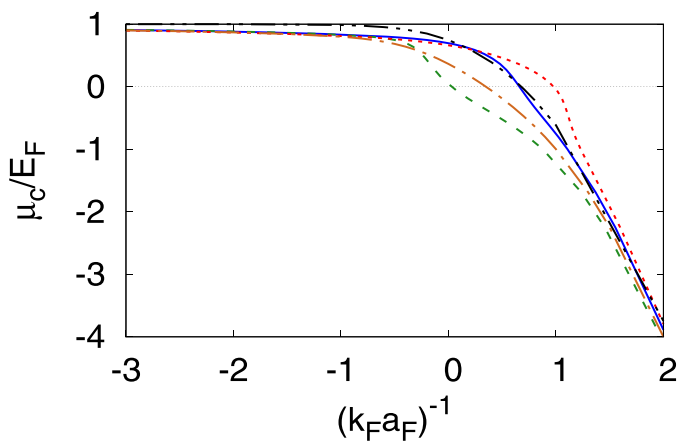

FIG. 7. (a) Five different approximations for the critical temperature $T_{c}$ are shown vs the coupling $\left(k_{F} a_{F}\right)^{-1}: T_{c}^{\text {(GMB+Popov) }}$ (solid line), $T_{c}^{\text {(GMB) }}$ (dashed line), $T_{c}^{\text {(Popov) }}$ (dotted line), $T_{c}^{(t \text {-matrix) }}$ (dash-dotted line), and $\bar{T}_{c}^{(\mathrm{BCS})}$ at the mean-field level (dashed double-dotted line). The inset compares the coupling dependence of $T_{c}^{\text {(GMB+Popov) }}$ (solid line) and of $T_{c}$ obtained in Refs. [33,34] within the self-consistent $t$-matrix approximation (dashed line). (b) The corresponding values of the chemical potential $\mu_{c}$ evaluated at $T_{c}$ are shown with the same conventions of panel (a). Both $T_{c}$ and $\mu_{c}$ are in units of the Fermi energy, $E_{F}$.

axis. Consistently with this observation, all curves (i)-(iv) have a maximum with the same height, although shifted at different couplings. On the other hand, this maximum is absent when the self-consistent $t$-matrix approximation of Refs. [33,34] is adopted to calculate $T_{c}$, as shown in the inset of Fig. 7(a) (dashed line), where a comparison with our most complete result $T_{c}^{(\mathrm{GMB}+\text { Popov) }}$ is also reported (solid line). Note that these two curves cross each other at about unitarity.

Quite generally, the presence of a maximum in the curve of the critical temperature vs coupling throughout the BCS-BEC crossover would be required by a general argument that, when approaching the extreme BEC limit where composite bosons can be treated as pointlike for all practical purposes, the BoseEinstein condensation temperature $T_{\mathrm{BEC}}$ for noninteracting bosons should be approached from above as shown in Ref. [35].

Figure 7(b) reports the results for the chemical potential $\mu_{c}$ associated with the values of the critical temperature $T_{c}$ corresponding to the approximations of Fig. 7(a). Note, in particular, that the BCS result for $\mu_{c}$ (dashed double-dotted line) corresponds to the noninteracting value taken at the temperature $T_{c}$. Note also that our most complete result for $\mu_{c}$, obtained by including both $\Sigma_{\mathrm{GMB}}^{\mathrm{B}}$ and $\Sigma_{\text {Popov }}^{\mathrm{B}}$ (solid line), gives

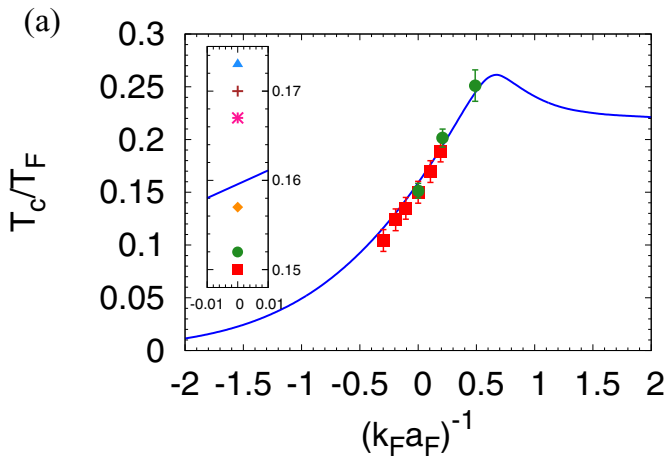

(b)

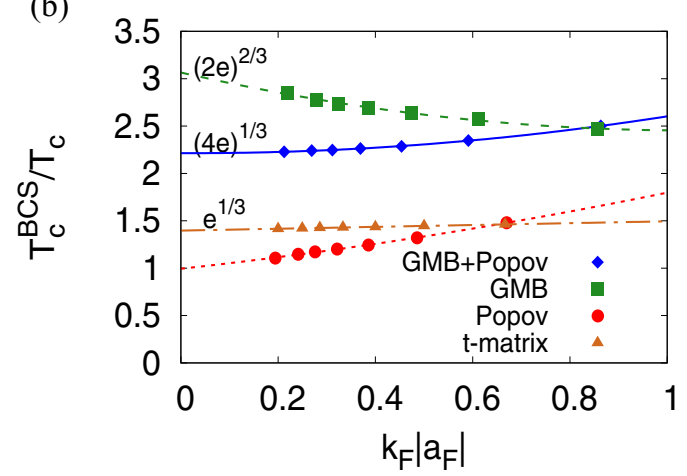

FIG. 8. (a) The results for the critical temperature $T_{c}^{\text {(GMB+Popov) }}$ (solid line) are compared with the quantum Monte Carlo data from Ref. [36] (squares with error bars) and Ref. [37] (dots with error bars) over an extended region of coupling. The inset focuses on the results at unitarity, by comparing our value of $T_{c}^{\text {(GMB+Popov) }}$ (solid line) with the results from Refs. [36-41] (corresponding to the symbols from bottom to top). (b) The ratio $T_{c}^{(\mathrm{BCS})} / T_{c}$ is shown in the BCS (weak-coupling) regime $k_{F}\left|a_{F}\right| \lesssim 1.0$ (with $a_{F}<0$ ), for the various approximations for $T_{c}$ (GMB+Popov, GMB, Popov, and $t$-matrix) reported in Fig. 7(a) over a more extended region of coupling. Here, $T_{c}^{(\mathrm{BCS})}$ is given by expression (2) that holds in the BCS regime.

larger values with respect to the (non-self-consistent) $t$-matrix approximation (dash-dotted line), while the self-consistent $t$-matrix approximation of Refs. $[33,34]$ gives a smaller value for $\mu_{c}$ at unitarity. We comment more extensively on this issue in Sec. IV.

Figure 8(a) compares our most complete results (GMB + Popov) for $T_{c}$ over an extended region of the coupling parameter $\left(k_{F} a_{F}\right)^{-1}$ with the quantum Monte Carlo (QMC) data available from Refs. [36,37]. The agreement between our results and the QMC data appears quite remarkable, taking into account the fact that our calculations contain no fitting parameters. It is also worth noting that the value of $T_{c}$ for $\left(k_{F} a_{F}\right)^{-1}=+0.5$ from Ref. [37] is larger than the BEC value $T_{\mathrm{BEC}}$ attained by our calculation in the extreme BEC limit, thus supporting the presence of a maximum in the curve of $T_{c} \mathrm{vs}$ $\left(k_{F} a_{F}\right)^{-1}$. In addition, the inset of Fig. 8(a) compares our result for $T_{c}$ at unitarity with the corresponding data reported both in experimental [38-40] and theoretical (QMC) [36,37,41] works. Also in this case, it is remarkable that our value for $T_{c}$ lies well within the boundaries provided by these data. 
Finally, Fig. 8(b) considers the ratio of the BCS critical temperature $T_{c}^{(\mathrm{BCS})}$ given by Eq. (2), which well approximates the mean-field result for $T_{c}$ in the BCS (weak-coupling) regime $k_{F}\left|a_{F}\right| \lesssim 1.0$ with $a_{F}<0$, with our numerical results for $T_{c}$ that were reported in Fig. 7(a) over a more extended region of coupling. The numerical results (symbols) have been extrapolated to the limit $k_{F}\left|a_{F}\right| \rightarrow 0$ through the fittings curves also reported in the figure (lines), where the limiting values coincide in each case with those obtained analytically in Secs. II C and IIE. This represents a further strong check on the accuracy of our calculations.

\section{Relevance of the dependence on wave vector and frequency of the pair propagators entering the GMB bosonic self-energy}

We have emphasized in the Introduction that this paper includes the full wave-vector and frequency dependence of the pair propagator $\Gamma_{0}$ in the calculation of the GMB contribution. We have also shown throughout this work that taking into account this dependence is essential for a correct calculation of the GMB contribution away from the (extreme) BCS regime. In this context, the question naturally arises about which one of these two dependencies (that is, wave vector or frequency) is the dominant one.

To answer this question, we have performed additional "partial calculations" of the GMB bosoniclike self-energy $\Sigma_{\mathrm{GMB}}^{\mathrm{B}}$, where the dependence on either the wave vector or the frequency has been neglected in both pair propagators $\Gamma_{0}$ that appear therein [while using the same values of $\left(\bar{T}_{c}, \bar{\mu}_{c}\right)$ in all calculations]. Specifically, in each $\Gamma_{0}$ we have the following: (i) Either we set the magnitude of the wave vectors $|\mathbf{p}|$ and $|\mathbf{q}|$ equal to $\sqrt{2} k_{\mu}$ (where the absolute value of the corresponding integrand is a maximum) while maintaining the full frequency dependence, (ii) or we set the frequency alternatively equal to zero or to $E_{F}$ while maintaining the full wave-vector dependence. The results of these calculations are reported in Fig. 9 on the BCS side of unitarity, where they are compared with the full calculation with both the wave-vector and frequency dependencies of $\Gamma_{0}$ taken into account. From this comparison one concludes that in the calculation of $\Sigma_{\mathrm{GMB}}^{\mathrm{B}}$, not only does the frequency dependence play a dominant role

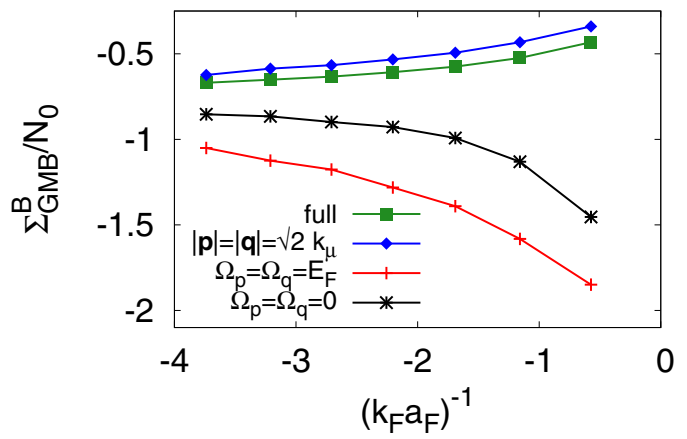

FIG. 9. The full calculation of $\Sigma_{\mathrm{GMB}}^{\mathrm{B}}$ vs $\left(k_{F} a_{F}\right)^{-1}$ is compared with partial calculations of the same quantity, where either the wavevector or the frequency dependence has been neglected in both pair propagators $\Gamma_{0}$ entering the expression of $\Sigma_{\mathrm{GMB}}^{\mathrm{B}}$. The pairs $\left(\bar{T}_{c}, \bar{\mu}_{c}\right)$ obtained by the full calculation are also used in the partial calculations. over the wave-vector dependence of $\Gamma_{0}$, but also neglecting the frequency dependence of $\Gamma_{0}$ yields a result considerably different from that of the full calculation, even with the wrong curvature of $\Sigma_{\mathrm{GMB}}^{\mathrm{B}}$ as a function of coupling.

\section{CONCLUDING REMARKS AND PERSPECTIVES}

In this paper, we have dealt with the calculation of the GMB correction in such a way that we could consistently extend it to scan the whole BCS-BEC crossover, whereby largely overlapping Cooper pairs on the BCS side evolve continuously into dilute composite bosons on the BEC side. The GMB correction was, in fact, originally introduced [2] to complement the BCS theory of superconductivity [1], which at that time was meant to apply only to largely overlapping Cooper pairs. For this reason, the GMB theory took advantage of specific approximations which are valid under these circumstances, resulting into a sizable reduction (by a factor of 2.2) of the value of the critical temperature with respect to the standard BCS value. The recent advent of accurate experiments with ultracold Fermi gases has then brought up the need for an accurate calculation of the GMB correction throughout the BCS-BEC crossover, thereby avoiding those approximations that would apply specifically to the BCS limit but could not be extended to the whole crossover. Previous attempts by diagrammatic methods to extend the calculation of the GMB correction throughout the BCS-BEC crossover $[13,14]$ apparently did not realize this delicate point and made an incorrect use of the same main approximations utilized in the original GMB paper.

Our handling of the GMB correction throughout the BCS$\mathrm{BEC}$ crossover has helped clarify an important physical point, that is, that the effective disentanglement between the particleparticle excitations (which are characteristic of superconductivity) and the particle-hole excitations (which are characteristic of screening) holds only in the (extreme) BCS limit of the crossover, which was of exclusive interest to the original GMB approach [2]. We have shown that, consistently with the key ingredients on which any physical sensible theoretical treatment of the BCS-BEC crossover must rely on, the above disentanglement between particle-particle and particle-hole excitations is not bound to occur when moving away from the BCS limit. In this context, we have also shown (cf. Appendix B) that the GMB correction, apart from maintaining its role in the BCS limit, acquires also an important role in the BEC limit of the crossover where it contributes significantly to the value of the scattering length $a_{B}$ of composite bosons. Otherwise, if one would (incorrectly) stick to maintaining the disentanglement between particle-particle and particle-hole excitations even in the BEC limit, the GMB correction would become totally irrelevant in this limit.

From the computational side, we have performed a very accurate numerical calculation of the GMB correction, maintaining the full dependence on wave vector and frequency of the pair propagators that appear in its expression. In this respect, we have been able to reproduce in a totally numerical fashion the factor of 2.2 for the reduction of the critical temperature with respect to the standard BCS value (while originally this result was obtained in an analytic way [2]). The accuracy of our numerical calculations was further tested against the analytic results that can be obtained both in the BCS and BEC 
limits of the crossover. To this end, we found it necessary to complement the GMB correction by a further correction based on the Popov theory for pointlike bosons, which provides an important mean-field shift contribution to the chemical potential of the constituent fermions in the BCS limit and to the chemical potential of the composite bosons in the BEC limit.

A definite success of our accurate simultaneous numerical handling of the GMB and Popov corrections throughout the BCS-BEC crossover is represented by the remarkable agreement we have obtained, between our calculated values of the critical temperature and those available by QMC calculations in the core of the crossover region (whereby $\left.-0.5 \lesssim\left(k_{F} a_{F}\right)^{-1} \lesssim+0.5\right)$ and by experiments with ultracold Fermi gases at unitarity. This agreement appears particularly significant, in light of the fact that our first-principles calculations do not contain any fitting parameter.

In this context, however, it should be pointed out that our calculation does not match the value of the chemical potential $\mu_{c}$ at $T_{c}$ and unitarity, within the range determined by an available experiment and by alternative theoretical calculations. Specifically, at unitarity and at the respective value of $T_{c}, \mu_{c} / E_{F}=0.3659$ within the non-self-consistent $t$-matrix approximation, 0.6971 by adding to it the GMB and Popov corrections as was done in the present work, 0.394 within the self-consistent $t$-matrix approximation of Refs. [33,34], and 0.42 as obtained experimentally in Ref. [39]. It thus appears that, adding the GMB and Popov corrections on top of the nonself-consistent $t$-matrix approximation makes the agreement with the experimental chemical potential worse than that obtained with the non-self-consistent $t$-matrix approximation itself.

This failure in obtaining a reasonable value of the thermodynamic chemical potential can be attributed to the lack of (at least some degree of) self-consistency in the fermionic propagators $G_{0}$ entering the diagrammatic structures of $\Sigma_{\text {Popov }}^{\mathrm{B}}$ [cf. Eq. (13)] and $\Sigma_{\mathrm{GMB}}^{\mathrm{B}}$ [cf. Eq. (20)], as well as of $\Gamma_{0}$ [cf. Eqs. (4) and (5)] and of the fermionic self-energy $\Sigma$ [cf. Eq. (7)]. To estimate the effect of introducing this self-consistency on the critical temperature obtained by including the Popov and GMB corrections, we can parallel the way the self-consistency was implemented in this paper for the bosoniclike pair propagator $\Gamma_{0}$. To this end, self-consistency in the fermionic propagators can approximately be dealt with at any given coupling by including a suitable constant (that is, independent of the fourvector $k$ ) self-energy shift $\Sigma_{0}$ in each of the above propagators $G_{0}$. Accordingly, this constant shift $\Sigma_{0}$ would get subtracted from the chemical potential $\mu$, such that $\mu \rightarrow \mu-\Sigma_{0}=\mu^{\prime}$. The only way this replacement would not affect the values of the critical temperature, as determined above within the full theory that includes $\Sigma_{\text {Popov }}^{\mathrm{B}}$ and $\Sigma_{\mathrm{GMB}}^{\mathrm{B}}$ [cf. the solid line in Fig. 7(a)], would be that $\mu^{\prime}$ thus determined corresponds to the chemical potential reported in Fig. 7(b) (solid line). An important observation can be made at this point, that the same value of $\mu^{\prime}$ also identifies an underlying Fermi surface with radius $k_{\mu^{\prime}}=\sqrt{2 m \mu^{\prime}}$ when $\mu^{\prime}>0$, at whose location a backbending occurs in the dispersion relation $\sqrt{\left(\frac{\mathbf{k}^{2}}{2 m}-\mu^{\prime}\right)^{2}+\tilde{\Delta}^{2}}$ as obtained from the single-particle spectral function, where $\tilde{\Delta}$ can be identified with a pairing gap in the superfluid phase [42] or with a pseudogap in the normal phase [43]. Figure 10 shows

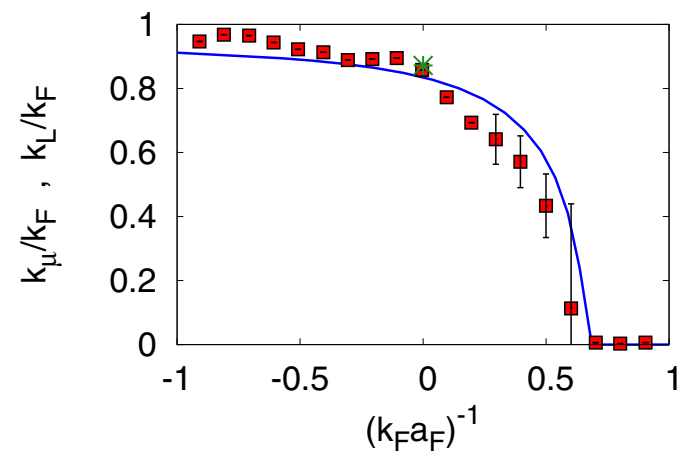

FIG. 10. The Luttinger wave vector $k_{L}$, obtained in Refs. [43,44] from the single-particle spectral function to identify the existence of an underlying Fermi surface in a Fermi gas with attractive interparticle interaction, is shown at $T_{c}$ vs coupling across the BCS-BEC crossover (squares with error bars). The corresponding value obtained at unitarity from the QMC calculation of Ref. [46] is also reported for comparison (star). This quantity is compared with the values of $k_{\mu}=\sqrt{2 m \mu}$ for $\mu>0$ (solid curve) obtained from the values of $\mu$ corresponding to the solid curve of Fig. 7(b).

the value of $k_{\mu^{\prime}}$ obtained in Refs. [43,44] (where it was referred to as the Luttinger wave vector $k_{L}$ ) from a study of the singleparticle spectral function at $T_{c}$ vs coupling (squares with error bars). These data are compared with the value of $k_{\mu}=\sqrt{2 m \mu}$ for $\mu>0$ (solid curve) obtained from the corresponding solid curve of Fig. 7(b). We recall that the values of $k_{L}$ reported in Fig. 10 have been validated by an extensive comparison with experiment over an extended range of coupling about unitarity $[43,45]$ and also by the value obtained at unitarity by the QMC calculation of Ref. [46] (identified by the star in Fig. 10). The quite good overall agreement obtained in this figure confirms our identification between $\mu$ of the present theory [the solid curve of Fig. 7(b)] with $\mu^{\prime}=\mu-\Sigma_{0}$ of a more refined theory that would include fermionic self-consistency, and supports our argument that the values of $T_{c}$ obtained in this paper by including the Popov and GMB corrections [the solid line in Fig. 7(a)] should not be affected by including explicitly this self-consistency.

A future natural extension of the present approach will be to consider the superfluid phase below $T_{c}$ and to calculate the pairing gap $\Delta$ down to zero temperature. Actually, this problem was considered in the original GMB paper [2], where it was found that at zero temperature also the value of $\Delta$ is reduced by a factor of 2.2 with respect to the BCS result. The challenge now would be to extend this GMB result to the whole BCS-BEC crossover for all temperatures between zero and $T_{c}$. To this end, a suitable diagrammatic form of the gap equation should be set up beforehand, which would allow us to take into account the GMB as well as the Popov correction extended to the superfluid phase. This topic will be postponed to future work.

Extending the proper treatment of the GMB correction to the superfluid phase across the BCS-BEC crossover appears particularly relevant at this time, since accurate experimental values of the pairing gap were recently made available via Bragg spectroscopy with ultracold Fermi gases [47]. This topic is also of much interest in the context of nuclear physics, where the value of the pairing gap was calculated at zero temperature 
by QMC methods essentially over the whole BCS side of unitarity [48].

\section{ACKNOWLEDGMENTS}

We are indebted to $S$. Girotti for help during the initial stage of this work. This work was partially supported by the Italian MIUR under PRIN-2015 Contract No. 2015C5SEJJ001.

\section{APPENDIX A: IMPLEMENTING THE NUMERICAL CALCULATION OF THE POPOV AND GMB DIAGRAMS}

We pass now to discuss in detail the procedures that we have adopted for implementing the numerical calculations of the Popov [Eq. (13)] and GMB [Eq. (20)] diagrammatic contributions (both with $Q=0$ ). A detailed discussion is relevant, in light of the fact that in these calculations we have included the full wave-vector and frequency dependence of the pair propagators $\Gamma_{0}$ appearing in expressions (13) and (20). In particular, this is especially important for the GMB expression (20), for which this inclusion was never attempted before. In the following, we discuss the two contributions separately.

For the sake of definiteness, the procedures to calculate numerically the integrals and sums entering the Popov and GMB diagrammatic contributions are discussed below in terms of the "bare" pair propagator $\Gamma_{0}$. In practice, however, these calculations are performed utilizing instead the "dressed" pair propagator $\Gamma$ of Eq. (44) in the place of $\Gamma_{0}$. This replacement will obviously not affect the procedures described below, which rest on taking full account of the wave-vector and frequency dependence of $\Gamma_{0}$ (or, equivalently, of $\Gamma$ ).

\section{Popov contribution}

For the sake of the following discussion, it is convenient to reproduce here expression (13) with $Q=0$ :

$$
\begin{aligned}
\Sigma_{\text {Popov }}^{\mathrm{B}}= & -2 \int \frac{d \mathbf{k}}{(2 \pi)^{3}} T \sum_{n} \int \frac{d \mathbf{q}}{(2 \pi)^{3}} T \sum_{v_{q}} \\
& \times G_{0}(k)^{2} G_{0}(-k) G_{0}(q-k) \Gamma_{0}(q)
\end{aligned}
$$

with the four-vector notation $k=\left(\mathbf{k}, \omega_{n}\right)$ and $q=\left(\mathbf{q}, \Omega_{v_{q}}\right)$, where $\omega_{n}$ and $\Omega_{v_{q}}$ are fermionic and bosonic Matsubara frequencies, respectively. Here, $G_{0}(k)=\left(i \omega_{n}-\xi_{\mathbf{k}}\right)^{-1}$ with $\xi_{\mathbf{k}}=\mathbf{k}^{2} /(2 m)-\mu$ and $\Gamma_{0}$ is given by Eq. (4). Expression (A1) contains summations over two Matsubara frequencies, and integrations over four angles (three of which turn out to be trivial) and over two magnitudes of wave vectors. We have optimized these sums and integrations by performing them in the following order.

Sum over the fermionic Matsubara frequency. The sum over the fermionic Matsubara frequency $\omega_{n}$ can be done analytically. One arrives at the following expression:

$$
\Sigma_{\mathrm{Popov}}^{\mathrm{B}}=-2 \int \frac{d \mathbf{k}}{(2 \pi)^{3}} \int \frac{d \mathbf{q}}{(2 \pi)^{3}} T \sum_{v_{q}} I\left(\xi_{\mathbf{k}}, \xi_{\mathbf{q}-\mathbf{k}} ; \Omega_{v_{p}}, \Omega_{v_{q}}\right) \Gamma_{0}(q),
$$

where

$$
\begin{aligned}
I\left(\xi_{\mathbf{k}}, \xi_{\mathbf{q}-\mathbf{k}} ; \Omega_{v_{p}}, \Omega_{v_{q}}\right)= & -\frac{f\left(\xi_{\mathbf{k}}\right)}{\left(2 \xi_{\mathbf{k}}\right)^{2}\left(\xi_{\mathbf{k}}+\xi_{\mathbf{q}-\mathbf{k}}-i \Omega_{v_{q}}\right)}-\frac{f\left(\xi_{\mathbf{k}}\right)}{2 \xi_{\mathbf{k}}\left(\xi_{\mathbf{k}}+\xi_{\mathbf{q}-\mathbf{k}}-i \Omega_{v_{q}}\right)^{2}}+\frac{d f\left(\xi_{\mathbf{k}}\right) / d \xi_{\mathbf{k}}}{2 \xi_{\mathbf{k}}\left(\xi_{\mathbf{k}}+\xi_{\mathbf{q}-\mathbf{k}}-i \Omega_{v_{q}}\right)} \\
& +\frac{f\left(-\xi_{\mathbf{k}}\right)}{\left(2 \xi_{\mathbf{k}}\right)^{2}\left(-\xi_{\mathbf{k}}+\xi_{\mathbf{q}-\mathbf{k}}-i \Omega_{v_{q}}\right)}-\frac{f\left(-\xi_{\mathbf{q}-\mathbf{k}}\right)}{\left(\xi_{\mathbf{k}}+\xi_{\mathbf{q}-\mathbf{k}}-i \Omega_{v_{q}}\right)^{2}\left(-\xi_{\mathbf{k}}+\xi_{\mathbf{q}-\mathbf{k}}-i \Omega_{v_{q}}\right)} .
\end{aligned}
$$

Angular integrations over the wave vectors. Expression (A3) depends only on the relative angle between $\mathbf{k}$ and $\mathbf{q}$. The integration over this angle is performed numerically with 100 points, while the integrations over the remaining three angles [which do not appear explicitly in expression (A3)] contribute a mere numerical factor $8 \pi^{2}$.

Radial integration over the fermionic wave vector. The radial integration over the magnitude $|\mathbf{k}|$ of the fermionic wave vector $\mathbf{k}$ is conveniently split into three intervals, namely, $\left[0, k_{c}\right],\left[k_{c}, k_{c}+2 k_{F}\right]$, and $\left[k_{c}+2 k_{F},+\infty\right]$, where

$$
k_{c}=\sqrt{2 m\left(\mu^{2}+T^{2}\right)^{1 / 2}}
$$

irrespective of the sign of $\mu$. In each interval, 50 integration points at most prove sufficient. The need for introducing the wave vector $k_{c}$ stems from the need for reproducing with good accuracy the shape of the peak developed by the integrand at about $k_{c}$. The second cutoff at $k_{c}+2 k_{F}$ is instead required to deal with the large- $|\mathbf{k}|$ tail of the integrand. [Similar considerations (about the peaks and tails of the integrands) apply when introducing the cutoffs $\tilde{q}_{c},\left(k_{c_{1}}, k_{c_{2}}\right),\left(q_{c_{1}}, q_{c_{2}}\right)$, and $\left(p_{c_{1}}, p_{c_{2}}\right)$ later on in this Appendix.]

Sum over the bosonic Matsubara frequency. It turns out that the last sum over the Matsubara frequency $\Omega_{v_{q}}$ decays like $\left|\Omega_{v_{q}}\right|^{-1.5}$ for large $\left|\Omega_{v_{q}}\right|$. This rather slow decay forces us to perform the sum with special care, by (i) summing up the discrete values from $v=0$ up to $v_{c_{1}}=500$, (ii) transforming the discrete sum into an integral from $\Omega_{v_{c_{1}}}$ up to $\Omega_{v_{c_{2}}}=1.5 \times$ $10^{6} E_{F}$, and (iii) calculating the integral analytically from $v_{c_{2}}$ up to infinity by estimating the coefficient of the $\left|\Omega_{v_{q}}\right|^{-1.5}$ powerlaw decay. (The sum for $\Omega_{v_{q}}<0$ can simply be obtained by complex conjugation.)

Radial integration over the bosonic wave vector. The last integral over $|\mathbf{q}|$ is divided into three intervals, namely, $\left[0, \tilde{q}_{c}\right]$, $\left[\tilde{q}_{c}, 3 \tilde{q}_{c}\right]$, and $\left[3 \tilde{q}_{c},+\infty\right]$, where $\tilde{q}_{c}=2 k_{c}$ with $k_{c}$ defined above in Eq. (A4). The integration over the first two intervals is performed numerically with 10 points in each interval, while the integration over the last interval is performed analytically by estimating the coefficient of the $|\mathbf{q}|^{-4}$ power-law decay. 


\section{GMB contribution}

It is again convenient to reproduce here expression (20) with $Q=0$ :

$$
\Sigma_{\mathrm{GMB}}^{\mathrm{B}}=\int \frac{d \mathbf{k}}{(2 \pi)^{3}} T \sum_{n} \int \frac{d \mathbf{p}}{(2 \pi)^{3}} T \sum_{v_{p}} \int \frac{d \mathbf{q}}{(2 \pi)^{3}} T \sum_{v_{q}} G_{0}(p-k) G_{0}(k-p) G_{0}(p+q-k) G_{0}(k) G_{0}(q-k) G_{0}(k-q) \Gamma_{0}(p) \Gamma_{0}(q)
$$

with the additional four-vector notation $p=\left(\mathbf{p}, \Omega_{v_{p}}\right)$ with respect to Eq. (A1), where $\Omega_{v_{p}}$ is a bosonic Matsubara frequency. Expression (A5) contains summations over three Matsubara frequencies and integrations over six angles (three of which turn out to be trivial) and over three magnitudes of wave vectors. We have optimized these sums and integrations by performing them in the following order.

Sum over the fermionic Matsubara frequency. It is convenient to perform the sum over the fermionic Matsubara frequency $\omega_{n}$ first. This can be done analytically in closed form, owing to the simple expression $G_{0}(k)=\left(i \omega_{n}-\xi_{\mathbf{k}}\right)^{-1}$ of the bare fermionic single-particle propagator. Otherwise, if one would dress the bare propagator $G_{0}$ with a fermionic self-energy $\Sigma$ [like in Eq. (8), or even in a more complicated fully self-consistent fashion], performing analytically the sum over $\omega_{n}$ would no longer be possible and one should unavoidably revert to a fully numerical evaluation of this sum.

In this way, one arrives at the compact expression

$$
\Sigma_{\mathrm{GMB}}^{\mathrm{B}}=\int \frac{d \mathbf{k}}{(2 \pi)^{3}} \int \frac{d \mathbf{p}}{(2 \pi)^{3}} T \sum_{v_{p}} \int \frac{d \mathbf{q}}{(2 \pi)^{3}} T \sum_{v_{q}} J\left(\xi_{\mathbf{k}}, \xi_{\mathbf{p}-\mathbf{k}}, \xi_{\mathbf{q}-\mathbf{k}}, \xi_{\mathbf{p}+\mathbf{q}-\mathbf{k}} ; \Omega_{v_{p}}, \Omega_{v_{q}}\right) \Gamma_{0}(p) \Gamma_{0}(q),
$$

where

$$
\begin{aligned}
& J\left(\xi_{\mathbf{k}}, \xi_{\mathbf{p}-\mathbf{k}}, \xi_{\mathbf{q}-\mathbf{k}}, \xi_{\mathbf{p}+\mathbf{q}-\mathbf{k}} ; \Omega_{v_{p}}, \Omega_{v_{q}}\right) \\
& =\frac{1}{2} \frac{1}{\left[\xi_{\mathbf{k}}+\xi_{\mathbf{p}+\mathbf{q}-\mathbf{k}}-i\left(\Omega_{v_{p}}+\Omega_{v_{q}}\right)\right]}\left\{-\frac{\mathcal{F}\left(\xi_{\mathbf{p}-\mathbf{k}}, \xi_{\mathbf{k}}, \Omega_{v_{p}}\right)}{\xi_{(-)} \xi_{(+)}}+\frac{\mathcal{F}\left(\xi_{\mathbf{q}-\mathbf{k}}, \xi_{\mathbf{k}}, \Omega_{v_{q}}\right)}{\xi_{(-)} \xi_{(+)}^{*}}-\frac{\mathcal{F}\left(-\xi_{\mathbf{p}-\mathbf{k}}, \xi_{\mathbf{k}}, \Omega_{v_{p}}\right)}{\xi_{(-)}^{*} \xi_{(+)}^{*}}\right. \\
& \quad+\frac{\mathcal{F}\left(-\xi_{\mathbf{q}-\mathbf{k}}, \xi_{\mathbf{k}}, \Omega_{v_{q}}\right)}{\xi_{(-)}^{*} \xi_{(+)}}+\frac{\mathcal{F}\left(\xi_{\mathbf{p}-\mathbf{k}},-\xi_{\mathbf{p}+\mathbf{q}-\mathbf{k}},-\Omega_{v_{q}}\right)}{\xi_{(-)} \xi_{(+)}}-\frac{\mathcal{F}\left(\xi_{\mathbf{q}-\mathbf{k}},-\xi_{\mathbf{p}+\mathbf{q}-\mathbf{k}},-\Omega_{v_{p}}\right)}{\xi_{(-)} \xi_{(+)}^{*}} \\
& \left.\quad+\frac{\mathcal{F}\left(-\xi_{\mathbf{p}-\mathbf{k}},-\xi_{\mathbf{p}+\mathbf{q}-\mathbf{k}},-\Omega_{v_{q}}\right)}{\xi_{(-)}^{*} \xi_{(+)}^{*}}-\frac{\mathcal{F}\left(-\xi_{\mathbf{q}-\mathbf{k}},-\xi_{\mathbf{p}+\mathbf{q}-\mathbf{k}},-\Omega_{v_{p}}\right)}{\xi_{(-)}^{*} \xi_{(+)}}\right\}
\end{aligned}
$$

and

$$
\xi_{( \pm)}=\xi_{\mathbf{p}-\mathbf{k}} \pm \xi_{\mathbf{q}-\mathbf{k}}+i\left(\Omega_{v_{p}}-\Omega_{v_{q}}\right), \quad \mathcal{F}(x, y, z)=\frac{f(x)-f(y)}{x(x-y+i z)}
$$

Depending on the values of $\Omega_{v_{p}}$ and $\Omega_{v_{q}}$, expression (A7) can be further simplified according to the following steps:

(i) When either $\Omega_{v_{p}} \neq 0$ or $\Omega_{v_{q}} \neq 0$, the eight terms therein can be reduced to four by the change of variable $\mathbf{k}^{\prime}=\mathbf{p}+\mathbf{q}-\mathbf{k}$ in half of the original terms.

(ii) When $\Omega_{v_{p}} \neq \Omega_{v_{q}}$, a further change of variables $p^{\prime}=q$ and $q^{\prime}=p$ in half of the four terms that are left after step (i) reduces them to two terms only.

(iii) When $\Omega_{v_{p}}=\Omega_{v_{q}} \neq 0$, one has instead to stick with the four terms obtained in step (ii).

(iv) Finally, when $\Omega_{v_{p}}=\Omega_{v_{q}}=0$, one has to stick with the original eight terms of expression (A7) in order to avoid introducing unnecessary principal values integrals in the numerical calculation.

Angular integrations over the wave vectors. Expressions (A7) and (A8) depend explicitly on three angles only. If one takes the $z$ axis in wave-vector space oriented along the direction of $\mathbf{k}$ and the $x$ axis such that $\mathbf{q}$ belongs to the $x-z$ plane, these three angles are the polar angle $\theta_{\mathbf{q}-\mathbf{k}}$ of $\mathbf{q}$ and the azimuthal $\varphi_{\mathbf{p}}$ and polar $\theta_{\mathbf{p}-\mathbf{k}}$ angles of $\mathbf{p}$. While the integration $\operatorname{over} \varphi_{\mathbf{p}}$ can be done analytically, the integrations over $\theta_{\mathbf{p}-\mathbf{k}}$ and $\theta_{\mathbf{q}-\mathbf{k}}$ have to be performed numerically. For both integrations 30 points prove usually sufficient. Finally, the integrations over the remaining three angles of $(\mathbf{k}, \mathbf{p}, \mathbf{q})$ [i.e., those on which expressions (A7) and (A8) do not depend] contribute a numerical factor $8 \pi^{2}$.

Radial integration over the fermionic wave vector. The radial integration over the magnitude $|\mathbf{k}|$ of the fermionic wave vector $\mathbf{k}$ is conveniently split into three intervals, namely, $\left[0, k_{c_{1}}\right],\left[k_{c_{1}}, k_{c_{2}}\right]$, and $\left[k_{c_{2}},+\infty\right]$. Here, $k_{c_{1}}=$ $\max \left\{k_{c},(|\mathbf{p}|+|\mathbf{q}|) / 2\right\}$ with $k_{c}$ given by Eq. (A4), while $k_{c_{2}}=$ $2 k_{c_{1}}$ for $\mu>0$ and $k_{c_{2}}=4 k_{c_{1}}$ for $\mu<0$. In each interval, 15 integration points prove sufficient.

Sum over the bosonic Matsubara frequencies. At this point, it is sufficient to calculate the sums over the bosonic Matsubara frequencies $\left(\Omega_{p}, \Omega_{q}\right)$ in the half plane $\Omega_{p} \geqslant 0$ only, since in the other half plane $\Omega_{p}<0$ the integrand can be obtained by complex conjugation. In this case, a natural cutoff is given by the frequency $\Omega_{c}=2 \pi v_{c} T=\left(|\mathbf{p}|^{2}+|\mathbf{q}|^{2}\right) /(2 m)$. The discrete sum over the frequencies $\left(\Omega_{p}, \Omega_{q}\right)$ is then computed over the trapezoidal area $0 \leqslant \Omega_{p} \leqslant \Omega_{c}$ and $-\left(\Omega_{c}+\Omega_{p}\right) \leqslant \Omega_{q} \leqslant$ $\Omega_{c}+\Omega_{p}$, while outside this area a continuum approximation 
is adopted which transforms the discrete sums into a twodimensional integral. Care should be exerted along the line $\Omega_{p}=\Omega_{q}$ where the integrand presents pronounced peaks. In the asymptotic regions $\Omega_{p} \gg \Omega_{c}$ and $\left|\Omega_{q}\right| \gg \Omega_{c}$ the integrand behaves like $\Omega_{p}^{-3.5}$ and $\left|\Omega_{q}\right|^{-3.5}$, such that 20 integration points prove sufficient for each variable.

Radial integrations over the bosonic wave vectors. First, the integral over $|\mathbf{q}|$ is divided in two intervals, namely, [0, $\left.q_{c_{1}}\right]$ and $\left[q_{c_{1}}, q_{c_{2}}\right]$, where $q_{c_{1}}=\max \left\{q_{c},|\mathbf{p}|\right\}$ and $q_{c_{2}}=7 k_{F}+|\mathbf{p}|$ with $q_{c}=\sqrt{2} k_{c}$ and $k_{c}$ given by Eq. (A4). The integrand turns out to have a minimum at about $q_{c_{1}}$, past which it decays to zero like $|\mathbf{q}|^{-3}$. It is found that the tail beyond $q_{c_{2}}$ contributes less than $1 \%$ to the final value of the integral and can thus be neglected.

Finally, the integral over $|\mathbf{p}|$ is also divided into two intervals, namely, $\left[0, p_{c_{1}}\right]$ and $\left[p_{c_{1}}, p_{c_{2}}\right]$, where $p_{c_{1}}=q_{c}$ while $p_{c_{2}}=5 k_{F}$ when $a_{F}<0$ and $p_{c_{2}}=7 k_{F}$ when $a_{F}>0$. Even in this case, the integrand turns out to have a minimum at about $p_{c_{1}}$, past which it decays to zero like $|\mathbf{p}|^{-4}$, while the tail beyond $p_{c_{2}}$ is found to contribute at most about $2-3 \%$ to the final value of the integral.

\section{APPENDIX B: CONTRIBUTION OF THE POPOV AND GMB DIAGRAMS TO THE SCATTERING LENGTH OF COMPOSITE BOSONS IN THE BEC LIMIT}

In Sec. II, the expressions of the Popov and GMB bosoniclike self-energies were obtained analytically in the BEC (strong-coupling) limit. We now show that these expressions are related to the scattering length $a_{B}$ for the low-energy scattering between composite bosons that form out of fermion pairs in the BEC limit. To this end, we rewrite the result in Eq. (19) for the Popov bosoniclike self-energy in the BEC limit in the form

$$
\Sigma_{\text {Popov }}^{\mathrm{B}}=-\frac{m k_{F}^{3} a_{F}^{2}}{6 \pi^{2}}=-\left(\frac{m^{2} a_{F}}{8 \pi}\right) \frac{8 \pi\left(2 a_{F}\right)}{2 m} \frac{n}{2},
$$

as well as the result in Eq. (40) for the GMB bosoniclike selfenergy in the BEC limit in the form

$$
\Sigma_{\mathrm{GMB}}^{\mathrm{B}}=\frac{16 \tilde{I}}{\pi^{2}} \frac{m k_{F}^{3} a_{F}^{2}}{6 \pi^{2}}=\left(\frac{m^{2} a_{F}}{8 \pi}\right) \frac{16 \tilde{I}}{\pi^{2}} \frac{8 \pi\left(2 a_{F}\right)}{2 m} \frac{n}{2} .
$$

Here, $2 m$ is the mass $m_{B}$ of a composite boson and $n / 2$ the density $n_{B}$ of the system of composite bosons.

In both expressions (B1) and (B2), the factor $m^{2} a_{F} /(8 \pi)$ is required to comply with the structure of the pair propagator (28), which in the (extreme) BEC limit acquires the polar form

$$
\Gamma_{0}\left(\mathbf{q}, \Omega_{q}\right)=-\left(\frac{8 \pi}{m^{2} a_{F}}\right) \frac{1}{i \Omega_{q}-\xi_{\mathbf{q}}^{B}} .
$$

Apart from the factor $-8 \pi /\left(m^{2} a_{F}\right)$, expression (B3) has the structure of the single-particle propagator of free (pointlike) bosons with dispersion relation $\xi_{\mathbf{q}}^{B}=\mathbf{q}^{2} /(4 m)-\mu_{B}$. In addition, when the interaction is taken into account in a lowdensity gas of (pointlike) bosons, this free-boson propagator is complemented by a self-energy of the form $8 \pi a_{B} n_{B} / m_{B}$ [18].

Upon translating this information back in the language of composite bosons, we then conclude that to the Popov
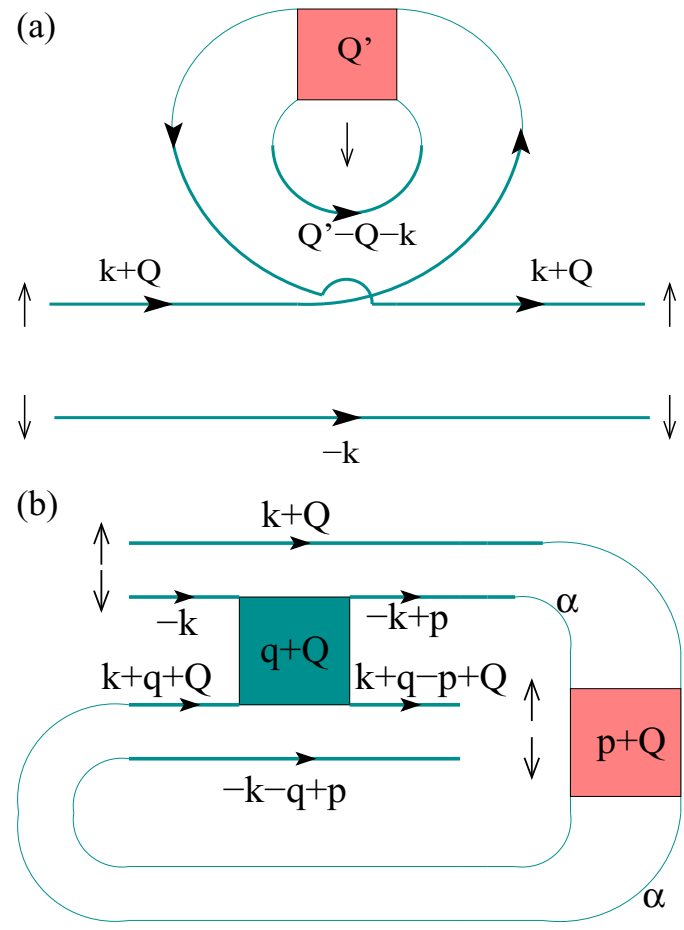

FIG. 11. Alternative drawing of (a) the Popov diagram of Fig. 2(a) and (b) the GMB diagram of Fig. 4(a), which in both cases evidences a substructure (in blue) contributing to the scattering length $a_{B}$ for composite bosons when $Q=0$ that survives in the limit $n \rightarrow 0$. To better highlight the correspondence with $a_{B}$, the fermionic spins have been explicitly indicated. The pair propagator $\Gamma_{0}$ responsible for the presence of a single factor of the density $n$ in both Eqs. (B1) and (B2) is also shown, in pink.

expression, Eq. (B1), there corresponds the value $a_{B}=2 a_{F}$ of the scattering length $a_{B}$ of composite bosons in terms of the scattering length $a_{F}$ of the constituent fermions. This value of $a_{B}$ amounts to treating two-fermion scattering at the level of the Born approximation [30]. The GMB expression, Eq. (B2), on the other hand, contributes the value $-\left(16 \tilde{I} / \pi^{2}\right) 2 a_{F} \simeq$ $-0.842 a_{F}$ to the scattering length $a_{B}$, with a different sign with respect to the Popov contribution. In conclusion, when combined together the Popov and GMB contributions alone yield the approximate value $a_{B} \simeq 1.158 a_{F}$, which has to be compared with the exact result $a_{B} \simeq 0.6 a_{F}$ obtained when all possible scattering processes among composite bosons are taken into account $[49,50]$.

In this context, it is instructive to identify directly in the Popov diagram of Fig. 2(a) and the GMB diagram of Fig. 4(a) the substructures, which are associated with the processes that contribute to the scattering length $a_{B}$ of composite bosons. This can be readily done by redrawing these diagrams in the alternative way shown in Fig. 11, which makes these processes evident. In addition, in Fig. 11(b) we identify the two fermionic propagators $G_{0}$ (by denoting them with the label $\alpha$ ), which would give rise to the particle-hole bubble in the BCS limit discussed in Sec. IIE, but which in the BEC limit here considered take part in the effective interaction between composite bosons. 
[1] J. Bardeen, L. N. Cooper, and J. R. Schrieffer, Theory of superconductivity, Phys. Rev. 108, 1175 (1957).

[2] L. P. Gorkov and T. M. Melik-Barkhudarov, Contribution to the theory of superfluidity in an imperfect Fermi gas, Sov. Phys. JETP 13, 1018 (1961) [Zh. Eksp. Teor. Fiz. 40, 1452 (1961)].

[3] V. M. Galitskii, The energy spectrum of a non-ideal Fermi gas, Sov. Phys. JETP 7, 104 (1958) [Zh. Eksp. Teor. Fiz. 34, 151 (1958)].

[4] C. A. Regal and D. S. Jin, Measurement of Positive and Negative Scattering Lengths in a Fermi Gas of Atoms, Phys. Rev. Lett. 90, 230404 (2003).

[5] J. R. Schrieffer, Theory of Superconductivity (Benjamin, New York, 1964).

[6] H. Heiselberg, C. J. Pethick, H. Smith, and L. Viverit, Influence of Induced Interactions on the Superfluid Transition in Dilute Fermi Gases, Phys. Rev. Lett. 85, 2418 (2000).

[7] R. Combescot, Trapped ${ }^{6} \mathrm{Li}$ : A High $T_{c}$ Superfluid? Phys. Rev. Lett. 83, 3766 (1999).

[8] D. S. Petrov, M. A. Baranov, and G. V. Shlyapnikov, Superfluid transition in quasi two-dimensional Fermi gases, Phys. Rev. A 67, 031601(R) (2003).

[9] M. A. Baranov, C. Lobo, and G. V. Shlyapnikov, Superfluid pairing between fermions with unequal masses, Phys. Rev. A 78, 033620 (2008).

[10] M. A. Resende, A. L. Mota, R. L. S. Farias, and H. Caldas, Finite temperature phase diagram of quasi-two-dimensional imbalanced Fermi gases beyond mean-field, Phys. Rev. A 86, 033603 (2012).

[11] D.-H. Kim, P. Törma, and J.-P. Martikainen, Induced Interactions for Ultracold Fermi Gases in Optical Lattices, Phys. Rev. Lett. 102, 245301 (2009).

[12] A. Bulgac, J. E. Drut, and P. Magierski, Spin 1/2 Fermions in the Unitary Regime: A Superfluid of a New Type, Phys. Rev. Lett. 96, 090404 (2006).

[13] Z.-Q. Yu, K. Huang, and L. Yin, Induced interaction in a Fermi gas with a BEC-BCS crossover, Phys. Rev. A 79, 053636 (2009).

[14] X.-X. Ruan, H. Gong, L. Du, W.-M. Sun, and H.-S. Zong, Effect of the induced interaction on the superfluid-transition temperature of ultracold Fermi gases within the T-matrix approximation, Phys. Rev. A 87, 043608 (2013).

[15] J. Lee and D-H. Kim, Induced interactions in the BCS-BEC crossover of two-dimensional Fermi gases with Rashba spinorbit coupling, Phys. Rev. A 95, 033609 (2017).

[16] S. Floerchinger, M. Scherer, S. Diehl, and C. Wetterich, Particlehole fluctuations in BCS-BEC crossover, Phys. Rev. B 78, 174528 (2008).

[17] P. Pieri and G. C. Strinati, Popov approximation for composite bosons in the BCS-BEC crossover, Phys. Rev. B 71, 094520 (2005).

[18] V. N. Popov, Functional Integrals and Collective Excitations (Cambridge University Press, Cambridge, U.K., 1987), Chap. 6.

[19] H.-J. Schulze, A. Polls, and A. Ramos, Pairing with polarization effects in low-density neutron matter, Phys. Rev. C 63, 044310 (2001).

[20] L. G. Cao, U. Lombardo, and P. Schuck, Screening effects in superfluid nuclear and neutron matter within Brueckner theory, Phys. Rev. C 74, 064301 (2006).

[21] C. A. R. Sá de Melo, M. Randeria, and J. R. Engelbrecht, Crossover from BCS to Bose Superconductivity: Transition
Temperature and Time-Dependent Ginzburg-Landau Theory, Phys. Rev. Lett. 71, 3202 (1993).

[22] P. Nozières and S. Schmitt-Rink, Bose condensation in an attractive fermion gas: From weak to strong coupling superconductivity, J. Low Temp. Phys. 59, 195 (1985).

[23] A. Perali, P. Pieri, G. C. Strinati, and C. Castellani, Pseudogap and spectral function from superconducting fluctuations to the bosonic limit, Phys. Rev. B 66, 024510 (2002).

[24] D. J. Thouless, Perturbation theory in statistical mechanics and the theory of superconductivity, Ann. Phys. 10, 553 (1960).

[25] Here and in the following, we refer to "bosoniclike self-energy" $\Sigma^{\mathrm{B}}$ as those diagrams in the particle-particle channel which dress the "bare" pair propagator $\Gamma_{0}$. For this reason, a prefactor $-m^{2} a_{F} /(8 \pi)$ has to be isolated from the expression of $\Sigma^{\mathrm{B}}$ to recover the corresponding bosonic expression, as shown explicitly in Appendix B.

[26] A. A. Abrikosov, L. P. Gorkov, and I. E. Dzyaloshinski, Methods of Quantum Field Theory in Statistical Physics (Dover, New York, 1963).

[27] C. J.Pethick and H. Smith, Bose-Einstein Condensation in Dilute Gases (Cambridge University Press, Cambridge, U.K., 2008), Chap. 16.

[28] G. D. Mahan, Many-Particle Physics (Kluwer, New York, 2000), Chap. 5.

[29] R. P. Feynman, An operator calculus having applications in quantum electrodynamics, Phys. Rev. 84, 108 (1951).

[30] P. Pieri and G. C. Strinati, Strong-coupling limit in the evolution from BCS superconductivity to Bose-Einstein condensation, Phys. Rev. B 61, 15370 (2000).

[31] A. L. Fetter and J. D. Walecka, Quantum Theory of ManyParticle Systems (McGraw-Hill, New York, 1971), Sec. 25.

[32] A. Perali, P. Pieri, L. Pisani, and G. C. Strinati, BCS-BEC Crossover at Finite Temperature for Superfluid Trapped Fermi Atoms, Phys. Rev. Lett. 92, 220404 (2004).

[33] R. Haussmann, Properties of a Fermi liquid at the superfluid transition in the crossover region between BCS superconductivity and Bose-Einstein condensation, Phys. Rev. B 49, 12975 (1994).

[34] R. Haussmann, W. Rantner, S. Cerrito, and W. Zwerger, Thermodynamics of the BCS-BEC crossover, Phys. Rev. A 75, 023610 (2007).

[35] G. Baym, J.-P. Blaizot, M. Holzmann, F. Laloë, and D. Vautherin, The Transition Temperature of the Dilute Interacting Bose Gas, Phys. Rev. Lett. 83, 1703 (1999).

[36] A. Bulgac, J. E. Drut, and P. Magierski, Quantum Monte Carlo simulations of the BCS-BEC crossover at finite temperature, Phys. Rev. A 78, 023625 (2008).

[37] E. Burovski, E. Kozik, N. Prokof'ev, B. Svistunov, and M. Troyer, Critical Temperature Curve in BEC-BCS Crossover, Phys. Rev. Lett. 101, 090402 (2008).

[38] S. Nascimbène, N. Navon, K. J. Jiang, F. Chevy, and C. Salomon, Exploring the thermodynamics of a universal Fermi gas, Nature (London) 463, 1057 (2010).

[39] M. J. H. Ku, A. T. Sommer, L. W. Cheuk, and M. W. Zwierlein, Revealing the superfluid lambda transition in the universal thermodynamics of a unitary Fermi gas, Science 335, 563 (2012).

[40] M. Horikoshi, S. Nakajima, M. Ueda, and T. Mukaiyama, Measurement of universal thermodynamic functions for a unitary Fermi gas, Science 327, 442 (2010). 
[41] O. Goulko and M. Wingate, Thermodynamics of balanced and slightly spin-imbalanced Fermi gases at unitarity, Phys. Rev. A 82, 053621 (2010).

[42] P. Pieri, L. Pisani, and G. C. Strinati, BCS-BEC crossover at finite temperature in the broken-symmetry phase, Phys. Rev. B 70, 094508 (2004).

[43] A. Perali, F. Palestini, P. Pieri, G. C. Strinati, J. T. Stewart, J. P. Gaebler, T. E. Drake, and D. S. Jin, Evolution of the Normal State of a Strongly Interacting Fermi Gas from a Pseudogap Phase to a Molecular Bose Gas, Phys. Rev. Lett. 106, 060402 (2011).

[44] F. Palestini and G. C. Strinati, Systematic investigation of the effects of disorder at the lowest order throughout the BCS-BEC crossover, Phys. Rev. B 88, 174504 (2013).

[45] J. P. Gaebler, J. T. Stewart, T. E. Drake, D. S. Jin, A. Perali, P. Pieri, and G. C. Strinati, Observation of pseudogap behaviour in a strongly interacting Fermi gas, Nat. Phys. 6, 569 (2010).
[46] J. Carlson and S. Reddy, Asymmetric Two-Component Fermion Systems in Strong Coupling, Phys. Rev. Lett. 95, 060401 (2005).

[47] S. Hoinka, P. Dyke, M. G. Lingham, J. J. Kinnunen, G. M. Bruun, and C. J. Vale, Goldstone mode and pair-breaking excitations in atomic Fermi superfluids, Nat. Phys. 13, 943 (2017).

[48] A. Gezerlis and J. Carlson, Low-density neutron matter, Phys. Rev. C 81, 025803 (2010).

[49] D. S. Petrov, C. Salomon, and G. V. Shlyapnikov, Scattering properties of weakly bound dimers of fermionic atoms, Phys. Rev. A 71, 012708 (2005).

[50] I. V. Brodsky, A. V. Klaptsov, M. Y. Kagan, R. Combescot, and $\mathrm{X}$. Leyronas, Bound states of three and four resonantly interacting particles, JETP Lett. 82, 273 (2005). 